Check for updates

Cite this: Mater. Chem. Front., 2020, 4, 1340

Received 15th January 2020, Accepted 21st February 2020

DOI: 10.1039/d0qm00029a

rsc.li/frontiers-materials

\section{Blue quantum dot-based electroluminescent light-emitting diodes}

\author{
Fei Chen, $\dagger^{a}$ Qingli Lin, $\dagger^{\mathrm{b}}$ Huaibin Shen (D) $*^{\mathrm{b}}$ and Aiwei Tang (D) $*^{a}$
}

Quantum dot light-emitting diodes (QD-LEDs) have been considered as one of the promising candidates for light sources in display applications owing to their good color saturation, high brightness, spectral tunability, and low processing cost. Thus far, the performance of red and green QD-LEDs has been significantly improved by optimizing QD materials and device architectures and by gaining a further understanding of their luminescence mechanism. However, as one of the three primary color sources, the performance of blue QD-LEDs still lags behind that of red and green QD-LEDs. Herein, we review the recent research progress in blue QD-LEDs, including Cd-based QD-LEDs, perovskite-based LEDs (PeLEDs), and heavy-metal-free QD-LEDs. The advances in Cd-based blue QD-LEDs are firstly introduced, and the efficiency has been improved from less than $0.1 \%$ to $\sim 20 \%$ through the optimization of the components and surface ligands of QDs as well as the optimization of device architecture and interfacial engineering, and the aging mechanism of blue QD-LEDs is also discussed. Next, we review PeLEDs based on different types of perovskite materials, including two-dimensional (2D)/quasi-2D perovskites included in organic-inorganic halide perovskites and all inorganic perovskites. Currently, state-of-the-art PeLEDs fabricated from quasi-2D organic-inorganic perovskites exhibit the maximum external quantum efficiency $\left(\eta_{\mathrm{EQE}}\right)$ exceeding $11 \%$. Furthermore, we summarize the research progress in heavy-metal-free blue QD-LEDs, which are fabricated from ZnSe, InP, Cu-based QDs and carbon dots. Although there is a huge gap in the device performance of heavy-metal-free LEDs as compared to the other two types of blue LEDs, the heavy-metal-free blue LEDs stand for a promising achievement toward their practical application. Finally, some challenges and possible development directions of blue QD-LEDs are suggested, which may speed up the further progress.

\section{Introduction}

Typically, semiconductor quantum dots (QDs) contain a few hundreds to tens of thousands of atoms and the diameter of the QDs is $\sim 1-20 \mathrm{~nm}$, which is smaller than the Bohr exciton radius (the distance between an electron and a hole). ${ }^{1}$ When the QD size is scaled to nanometers, the electronic energy level near the Fermi level splits from continuous into discrete energy levels, rendering the electronic structure, and optical and magnetic properties of materials tunable by varying the QD size. $^{2}$ Colloidal QDs have been a subject of active research in applications including solar cells, ${ }^{3,4}$ biological applications, ${ }^{5}$ photodetectors, ${ }^{6,7}$ and light-emitting diodes (LEDs) ${ }^{8-11}$ owing to their unique physical properties of size-dependent energy

\footnotetext{
${ }^{a}$ Key Laboratory of Luminescence and Optical Information, Ministry of Education, School of Science, Beijing Jiaotong University, Beijing 100044, China. E-mail: awtang@bjtu.edu.cn

${ }^{b}$ Key Laboratory for Special Functional Materials of Ministry of Education, Henan University, Kaifeng 475004, China. E-mail: shenhuaibin@henu.edu.cn $\dagger$ These authors contributed equally.
}

band gap, narrow spectral emission bandwidths, broad spectral photoresponse from ultraviolet to infrared, and compatibility with the solution process. In particular, QD-LEDs are a promising candidate for next-generation displays owing to their high color purity and color-tunable emission achieved by changing the size of the core. Thus far, red QD-LEDs with a maximum external quantum efficiency ( $\eta_{\mathrm{EQE}}$ ) of $20.5 \%$ and a device lifetime of more than $100000 \mathrm{~h}$ at $100 \mathrm{~cd} \mathrm{~m}^{-2}$ have been reported. ${ }^{12}$ In 2019 , by introducing compositional graded CdZnSe/ZnSe/ZnSeS/ZnS QDs with high photoluminescence (PL) quantum yields (QYs), suppressed nonradiative Förster resonant energy transfer and Auger recombination, and favorable valence band alignment for enhanced hole injection, Shen et al. demonstrated a hero $\eta_{\mathrm{EQE}}$ and current efficiency of $23.9 \%$ and $100.5 \mathrm{~cd} \mathrm{~A}^{-1}$, respectively, for green QD-LEDs. ${ }^{13}$ Using the interconnecting layer (ICL) proposed therein, all-solution-processed tandem green and red QD-LEDs were successfully demonstrated with an impressive $\eta_{\mathrm{EQE}}$ of over $42 \%$ and $34 \%,{ }^{14,15}$ respectively, which are the highest values ever reported and are comparable with those of state-of-the-art phosphorescent organic LEDs (OLEDs). Notably, the $\eta_{\mathrm{EQE}}$ of blue QD-LEDs has also increased from $\sim 0.1 \%$ to 
19.8\% because of their unique QD structure (i.e., the composition gradient in the thick shells), the stable surface modification of the QDs (i.e., appropriate surface ligands), the improved balance between hole and electron current (achieved using appropriate device architecture), and a deep understanding of the aging mechanism in blue QD-LEDs. ${ }^{16-18}$ However, the operational lifetime of these blue QD-LEDs is lower than that of the red and green QD-LEDs. ${ }^{19-21}$

Besides Cd-based QDs, perovskite nanocrystals (NCs) have also attracted scientific attention owing to their narrow fullwidth half maximum (FWHM) and high PL QYs. Currently, green perovskite-based LEDs (PeLEDs) exhibit a record $\eta_{\mathrm{EQE}}$ that surpasses $20 \%$ by mixing a presynthesized $\mathrm{CsPbBr}_{3}$ perovskite with a $\mathrm{CH}_{3} \mathrm{NH}_{3} \mathrm{Br}$ (MABr) additive. This enhanced device performance can be attributed to the formation of a $\mathrm{CsPbBr}_{3} / \mathrm{MABr}$ quasi-core/ shell structure that passivates the nonradiative defects present in $\mathrm{CsPbBr}_{3}$ crystals, boosting the PL quantum efficiency. ${ }^{22}$ The $\eta_{\mathrm{EQE}}$ of red PeLEDs has also exceeded $20 \%$ (21.3\%) through anion exchange from pristine $\mathrm{CsPbBr}_{3}$ using halide-anion-containing alkyl ammonium and aryl ammonium salts. ${ }^{23}$ Unfortunately, the $\eta_{\mathrm{EQE}}$ of blue PeLEDs is still less than $12 \%$. In the case of both Cd-based QDs and perovskite NCs, intrinsic toxicity limits further commercialization owing to the release of free $\mathrm{Cd}^{2+}$ or $\mathrm{Pb}^{2+}$ ions into the environment. This limitation paved the way for introducing heavy-metal-free QDs, wherein a maximum luminance of $4490 \mathrm{~cd} \mathrm{~m}^{-2}$ and a peak $\eta_{\mathrm{EQE}}$ of $7.8 \%$ of red QD-LEDs were reported using 1-dodecanethiol (DT) coated CIS/ZnS core/shell QDs as the emitting layer. ${ }^{24}$ In addition, for InP-based QDs, the peak $\eta_{\mathrm{EQE}}$ of red and green QD-LEDs also exceeded $12 \%$ and $13 \%$, respectively, through the optimization of the composition gradient in QD shells and the doping of the ZnO electron transport layer (ETL). ${ }^{25,26}$ Nevertheless, heavy-metal-free blue QD-LEDs have been rarely reported.

The poor performance of blue QD-LEDs may be attributed to the following phenomena: (1) low PL QYs of the blue QD film. Although QDs with high PL QYs have been synthesized, the PL QY of QD films has rapidly reduced owing to the energy transfer between QDs post purification and film formation. The low PL QY of the QD film results in a low recombination efficiency of electrons and holes in the emitting layer, leading to the low efficiency of the QD-LED. (2) Low charge-transfer efficiency between QDs in solid-state films. Typically, QDs include inorganic core/shell and surface organic ligands, which are necessary to determine the electrical properties of the QD layer. In general, previously, fatty acids with long hydrocarbon chains were chosen as surface ligands of colloidal QDs. Although these long-chain organic molecules can prevent QD aggregation in organic solvents and reduce QD surface defects, they also affect charge injection into QDs and the charge carrier transport within the QD layer by forming a dense insulating layer. ${ }^{27}$ Hence, selecting appropriate ligands that can not only stabilize the QDs and maintain their intrinsic good photoelectric properties but also realize efficient charge transfer between QDs in solid-state films is necessary. (3) The imbalance between the electron and hole injection in blue QD-LEDs. In the conventional organic-inorganic hybrid QD-LEDs, a large interface barrier exists between the QD emitting layer and the organic hole transport layer (HTL) formed by the deep valence band energy level for blue emission. ${ }^{28}$ However, research on component and shell thickness of blue QDs and appropriate choice of charge transport materials is currently insufficient. (4) Charge transfers across the QD-ZnO junction and charge accumulation in the ETL generate positively charged blue QDs and negatively charged $\mathrm{ZnO}$, reducing the efficiency of radiative recombination in the QD emitting layer. ${ }^{29}$ (5) The human eye is insensitive to blue light. Compared with $530 \mathrm{~nm}$-green light and $620 \mathrm{~nm}$-red light, only $1 / 9$ and $1 / 4$ of the radiated power, respectively, of blue light is needed to achieve the same visual effect.

Therefore, the device performance of blue QD-LEDs can be substantially improved by overcoming the following challenges: the low quality of blue QD-emitting materials and the inappropriate hole transport materials and device structure for blue emission. Bearing this in mind, the performance of blue QD-LEDs can be optimized by designing nanostructures with low defects, appropriate band-gap levels, and high PL QYs; modifying the surface of semiconductor QDs with appropriate ligands; and optimizing the interfaces and device architecture of blue devices. In this review, we aim to present recent advances in the improvement of blue device efficiency, including Cd-based blue QD-LEDs, blue PeLEDs, and heavy-metal-free QD-LEDs. We firmly believe that the performance of blue QD-LEDs will considerably improve in the near future and become comparable with that of their red and green counterparts.

\section{Cd-based blue QD-LEDs}

Although few blue QD-LEDs with a maximum brightness of $\sim 125 \mathrm{~cd} \mathrm{~m}^{-2}$ and a FWHM $\geq 32 \mathrm{~nm}$ have been demonstrated, their emission spectra inevitably exhibited significant tails extending into the green and red regions of the spectrum. ${ }^{8}$ Until 2007, bright and color-saturated emission from blue LEDs was reported by Tan, ${ }^{30}$ who structurally engineered core/shell CdS/ZnS QDs using low shell-synthesis temperature $\left(\sim 200{ }^{\circ} \mathrm{C}\right)$ (Fig. 1a). As shown in Fig. 1b and c, the device based on the core/shell QDs emitted pure blue light at $460 \mathrm{~nm}$ with a narrow FWHM of $18 \mathrm{~nm}$, and the peak luminance reached up to $1600 \mathrm{~cd} \mathrm{~m}^{-2}$. In particular, the long-wavelength tail of electroluminescence (EL) was minimized to less than $5 \%$ of the total emission. However, a large lattice mismatch of about $12 \%$ between CdS and ZnS causes interfacial strain, reducing the PL QY of the QDs. Therefore, Anikeeva et al. chose ZnCdSalloyed QD cores as blue-emitting QDs with the PL peak at $460 \mathrm{~nm}$ and a high PL QY of 50\%. Selecting spiro-TPD as the HTL and TPBi as the ETL, a peak $\eta_{\mathrm{EQE}}$ of $0.4 \%$ was obtained. ${ }^{31}$ TPBi, a small molecule deposited via vacuum deposition, has inferior thermal stability and is highly susceptible to degradation induced by oxygen and/or moisture. Hence, a solution-processable inorganic charge transport layer (CTL) is more desirable for stable and high-performance QD-LEDs. In 2011, there was a major breakthrough in the performance of QD-LEDs. Qian et al. synthesized crystalline ZnO nanoparticles (NPs) via a solution-precipitation 

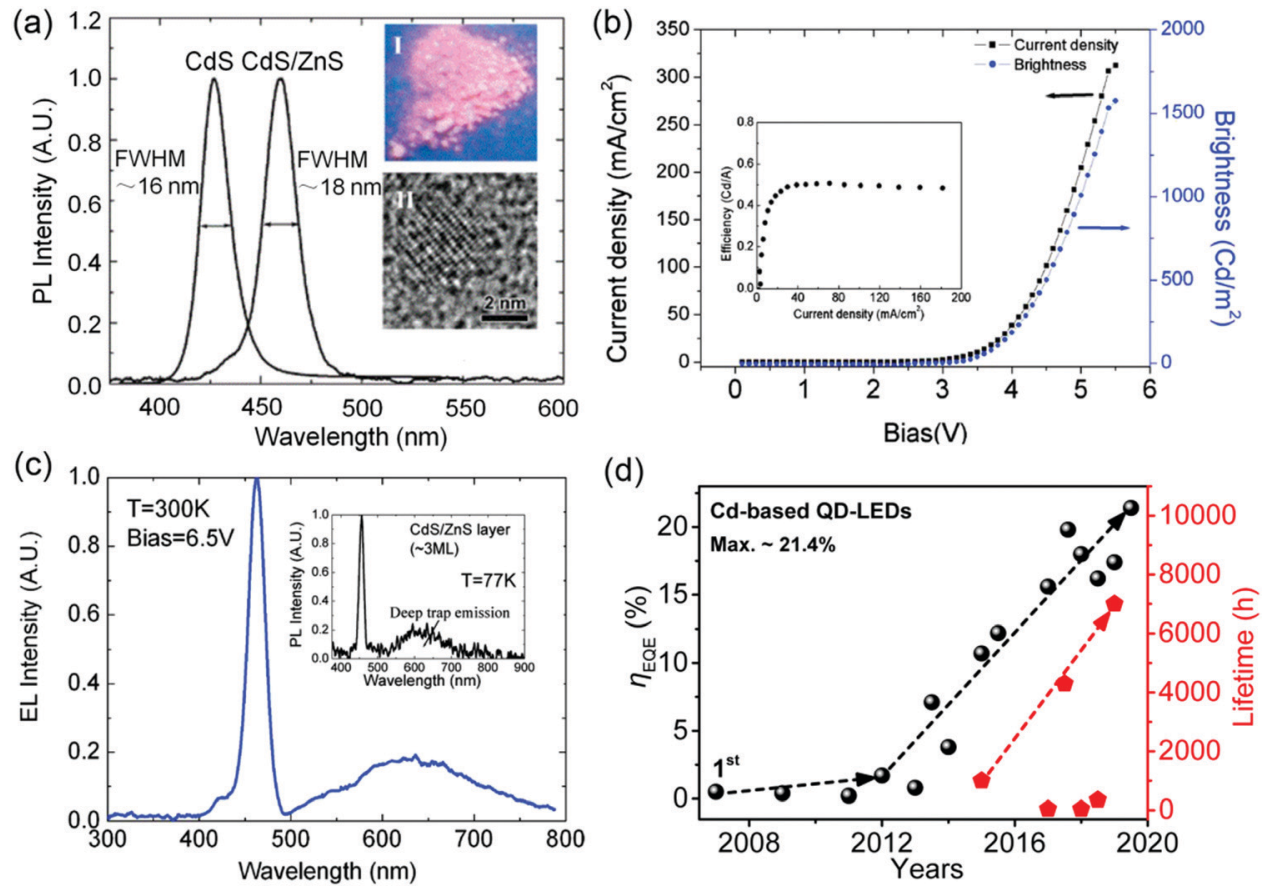

Fig. 1 (a) Photoluminescence (PL) spectra of CdS cores and core/shell CdS/ZnS QDs stored in tetrachloroethylene solvent. Inset I: Fluorescent image of QD power under UV light. Inset II: High-resolution transmission electron microscopy (HR-TEM) image of a core/shell CdS/ZnS QD. (b) J-L-V characteristics of a blue quantum dot light-emitting diode (QD-LED); the inset plots current efficiency vs. current density. (c) Electroluminescence (EL) spectrum of a QD-LED measured at $6.5 \mathrm{~V}$. Inset: PL spectrum of CdS/ZnS QDs in the LED active region measured at $77 \mathrm{~K}$. Reproduced with permission. ${ }^{30}$ Copyright 2007, American Chemical Society. (d) Progress of blue QD-LEDs in the faction of $\eta_{\text {EQE }}$ and operating lifetime at an initial luminance of $100 \mathrm{~cd} \mathrm{~m}^{-2}$.

method; these NPs adopted a wurtzite structure with an average diameter of $3 \mathrm{~nm}$. Using these ZnO NPs as the ETL, all-solutionprocessable multilayer QD-LEDs were fabricated, in which all other layers were sequentially deposited on ITO via spin-coating except for the Al cathode, which was deposited via vacuum thermal evaporation. The resultant devices demonstrated a maximum brightness of $4200 \mathrm{~cd} \mathrm{~m}^{-2}$ for blue light, which was the highest brightness value reported at that time. Notably, that device exhibited a rather low turn-on voltage of $2.4 \mathrm{~V}$, which was lower than the photon voltages due to a ZnO NP-induced Auger upconversion mechanism. ${ }^{32}$ As observed in Fig. 1d, since then, the $\eta_{\mathrm{EQE}}$ and lifetime of blue QD-LEDs have increased up to $19.8 \%$ and more than $7000 \mathrm{~h}$, respectively, owing to the nanostructure engineering and stable surface modification of the QD emitting layer, the optimization of related device architecture, and deep understanding of the aging mechanism in blue QD-LEDs. ${ }^{19,33}$ In this section, we will review the recent progress in Cd-based blue QD-LEDs, divided into three aspects based on various improvements. In addition, Table 1 summarizes the detailed device characteristics of blue Cd-based QD-LEDs.

\subsection{Nanostructure engineering and stable surface modification of the QD emitting layer}

A prerequisite for realizing high-performance QD-LEDs is to use a QD film having a high PL QY. Although blue-emitting QDs with high PL QYs have been synthesized, the PL QYs of such QD films rapidly decrease owing to the energy transfer between QDs post purification and film formation. Therefore, introducing intermediate shells, a composition-gradient in alloyed core/ shell structures, and increasing the shell thickness are required for the blue QD film to maintain its initial PL QY. For example, Shen et al. prepared high-quality blue-green-emitting quaternary ZnCdSSe/ZnS QDs by adjusting the ratios between $\mathrm{Zn}$ and $\mathrm{Cd}$ and Se and $S$ in the core. The PL QYs of as-synthesized ZnCdSSe/ZnS core/shell QDs can reach up to $50-75 \%$ with emissions between 450 and $550 \mathrm{~nm}$. However, the $\eta_{\mathrm{EQE}}$ of the related blue QD-LEDs was only $0.8 \%$, which may be due to the low PL QY of the blueemitting QDs. ${ }^{34}$ Lee et al. first reported highly fluorescent blue (452 nm) alloyed QDs of ZnCdS core/ZnS shell with a surprisingly high PL QY of $98 \%$ by optimizing the $\mathrm{ZnS}$ shelling time. Furthermore, although the $3 \mathrm{~h}$-shelled $\mathrm{ZnCdS} / \mathrm{ZnS}$ film exhibited a somewhat reduced PL QY of 86\%, PL spectra between the solidstate QD film and dilute QD solution nearly matched without any noticeable peak shift. The exceptional performance of $\mathrm{ZnCdS} / \mathrm{ZnS}$ QDs can be attributed to the following reasons. First, the long shelling duration could produce better surface passivation of ZnCdS core QDs with the ZnS shell, structurally improving the $\mathrm{ZnCdS} / \mathrm{ZnS}$ interface via effective thermal annealing. Second, the shelling temperature of $310{ }^{\circ} \mathrm{C}$, which is high enough to induce interfacial alloying, would render the core/shell boundary less abrupt, creating a relatively smooth interface in terms of composition. To verify the effectiveness of QDs with a thick shell, they fabricated QD-LEDs (Fig. 2a) by sequentially spin-coating the solutions of PEDOT:PSS and PVK in chlorobenzene, ZnCdS/ZnS QDs in hexane, and ZnO NPs in ethanol; consequently, an excellent $\eta_{\mathrm{EQE}}$ of $7.1 \%$ and a high luminance of $2624 \mathrm{~cd} \mathrm{~m}^{-2}$ 
Table 1 Recent advances of blue Cd-based QD-LEDs

\begin{tabular}{|c|c|c|c|c|c|c|c|c|}
\hline Year & Device structure & $\begin{array}{l}T_{50} \\
(\mathrm{~h})\end{array}$ & $\begin{array}{l}\text { PL peak } \\
(\mathrm{nm})\end{array}$ & $\begin{array}{l}\eta_{\mathrm{EQE}} \\
(\%)\end{array}$ & $\begin{array}{l}\eta_{\mathrm{A}} \\
\left(\mathrm{cd} \mathrm{\textrm {A } ^ { - 1 }}\right)\end{array}$ & $\begin{array}{l}V_{\text {on }} \\
(\mathrm{V})\end{array}$ & $\begin{array}{l}L_{\max } \\
\left.(\mathrm{cd} \mathrm{m})^{-2}\right)\end{array}$ & Ref. \\
\hline 2007 & ITO/PEDOT:PSS/poly-TPD/CdS/ZnS/Al & - & 460 & - & 0.506 & 2.5 & 1600 & 30 \\
\hline 2009 & ITO/PEDOT:PSS/spiro-TPD/ZnCdS/ZnS/TPBi/Al & - & 460 & 0.4 & - & - & - & 31 \\
\hline 2011 & ITO/PEDOT:PSS/poly-TPD/CdSe/ZnS/ZnO/Al & - & 470 & 0.22 & 0.32 & 2.4 & 4200 & 32 \\
\hline 2012 & ITO/ZnO/CdZnS@ZnS/CBP/ $\mathrm{MoO}_{3} / \mathrm{Al}$ & - & 433 & 1.7 & 0.4 & 3.0 & 2250 & 50 \\
\hline 2013 & ITO/PEDOT:PSS/TFB/Zn ${ }_{x} \mathrm{Cd}_{1-x} \mathrm{~S}_{y} \mathrm{Se}_{1-y} / \mathrm{ZnS} / \mathrm{ZnO} / \mathrm{Al}$ & - & 460 & 0.8 & 0.52 & 2.4 & 4700 & 34 \\
\hline 2013 & ITO/PEDOT:PSS/PVK/ZnCdS/ZnS/ZnO/Al & - & 452 & 7.1 & 2.2 & 4.0 & 2624 & 35 \\
\hline 2014 & ITO/PEDOT:PSS/TFB/Zn ${ }_{x} \mathrm{Cd}_{1-x} \mathrm{~S} / \mathrm{ZnS} / \mathrm{ZnO} / \mathrm{Al}$ & - & 445 & 3.8 & 1.13 & 3.8 & 4100 & 36 \\
\hline 2014 & ITO/AZO:Cs ${ }_{2} \mathrm{CO}_{3} / \mathrm{CdS} / \mathrm{ZnS} /$ poly-TPD/PEDOT:PSS/Al & - & - & - & 0.06 & 3.6 & 249 & 52 \\
\hline 2015 & ITO/PEDOT:PSS/PVK/Cd ${ }_{1-x} \mathrm{Zn}_{x} \mathrm{~S} @ \mathrm{ZnS} / \mathrm{ZnO} / \mathrm{Al}$ & 1000 & 455 & 10.7 & 4.4 & 2.6 & 4000 & 20 \\
\hline 2015 & ITO/PEDOT:PSS/TFB/ZnCdS/ZnS-OT/ZnO/Al & - & 441 & 12.2 & 1.9 & 2.6 & 7600 & 38 \\
\hline 2017 & ITO/PEDOT:PSS/PVK/Zn ${ }_{x} \mathrm{Cd}_{1-x} \mathrm{~S} / \mathrm{ZnS} / \mathrm{ZnO} / \mathrm{Al}$ & - & 445 & 15.6 & 1.71 & 5.8 & 4500 & 39 \\
\hline 2017 & ITO/PEDOT:PSS/PVK/CdSe/ZnS/ZnO/Al & 47.5 & 466 & 19.8 & 14.1 & 5.1 & 4890 & 33 \\
\hline 2017 & PET/ITO-Ag-ITO/ZnO/CdSe/ZnS/mCP/MoO $/ \mathrm{Ml}$ & - & 478 & - & 16.1 & 2.8 & 18127 & 55 \\
\hline 2018 & $\mathrm{ITO} / \mathrm{ZnO} / \mathrm{CdZnSe} / \mathrm{ZnS} / \mathrm{mCP} / \mathrm{MoO}_{3} / \mathrm{Al}$ & - & 455 & 3.23 & 1.08 & 3.1 & - & 37 \\
\hline 2018 & ITO/PEDOT:PSS/PVK/ZnCdS/Cd ${ }_{x} \mathrm{Zn}_{1-x} \mathrm{~S} / \mathrm{ZnS} / \mathrm{ZnO} / \mathrm{Al}$ & 47.4 & 445 & 18.0 & 3.4 & 6.5 & 6768 & 40 \\
\hline 2018 & ITO/PEDOT:PSS/TFB/ZnCdSe/ZnS//ZnS/PMMA/ZnO/Al & 355 & 472 & 16.2 & 11.8 & 2.5 & 14100 & 41 \\
\hline 2018 & ITO/PEDOT:PSS/poly-TPD/DNA/ZnCdS/ZnS/ZnO/Al & - & 462 & 5.65 & 2.3 & 3.2 & 16655 & 43 \\
\hline 2018 & ITO/PEDOT:PSS/PVK:TPD/ZnCdS/ZnS/ZnO/Ag & - & 457 & 8.62 & 6.90 & 3.1 & 10824 & 44 \\
\hline 2018 & ITO/PEDOT:PSS/PVK/ZnCdSeS/ZnS/Al:ZnO/Al & - & 467 & 4.0 & 2.7 & 3.8 & - & 48 \\
\hline 2018 & ITO/PEIE/ZnO/ZnCdS/ZnS/PVK/MoO $/ \mathrm{Al}$ & - & 458 & 5.5 & 2.5 & 4.2 & 2640 & 51 \\
\hline 2018 & ITO/ZnO/CdSe/ZnS/PVK/TFB/PMAH/Al & - & 454 & 5.99 & 1.99 & 4.1 & 4140 & 53 \\
\hline 2019 & ITO/PEDOT:PSS/TFB/CdSe/ZnSe/ZnO/Al & 7000 & 476 & 8.05 & - & 2.5 & 62600 & 19 \\
\hline 2019 & ITO/PEDOT:PSS/TFB:TCTA/ZnCdS/ZnS/AZO:ZrAcac/Al & - & 464 & 10.7 & 5.7 & 3.0 & 34874 & 45 \\
\hline 2019 & ITO/PEDOT:PSS/PVK/CdSe/ZnS/LiF/ZnO/Al & - & 465 & 9.8 & 7.9 & 3.3 & 36970 & 46 \\
\hline 2019 & ITO/PEDOT:PSS/TFB/ZnCdS/ZnS/TBS-PBO/ZMO/Al & - & 449 & 17.4 & - & 3.0 & 7992.5 & 47 \\
\hline 2019 & ITO/PEDOT:PSS/poly-TPD/ZnCdS/ZnS/ZnO/Al: $\mathrm{Al}_{2} \mathrm{O}_{3}$ & - & 452 & 8.92 & 3.29 & 3.4 & 27753 & 49 \\
\hline 2019 & ITO/PEDOT:PSS/PVK/CdZnSeS/ZnS/ZMO/Al/HATCN/MoO ${ }_{3} / \mathrm{PVK} / \mathrm{CdZnSeS/ZnS/ZMO/Al}$ & - & 474 & 21.4 & 17.9 & 8.8 & 26800 & 54 \\
\hline
\end{tabular}
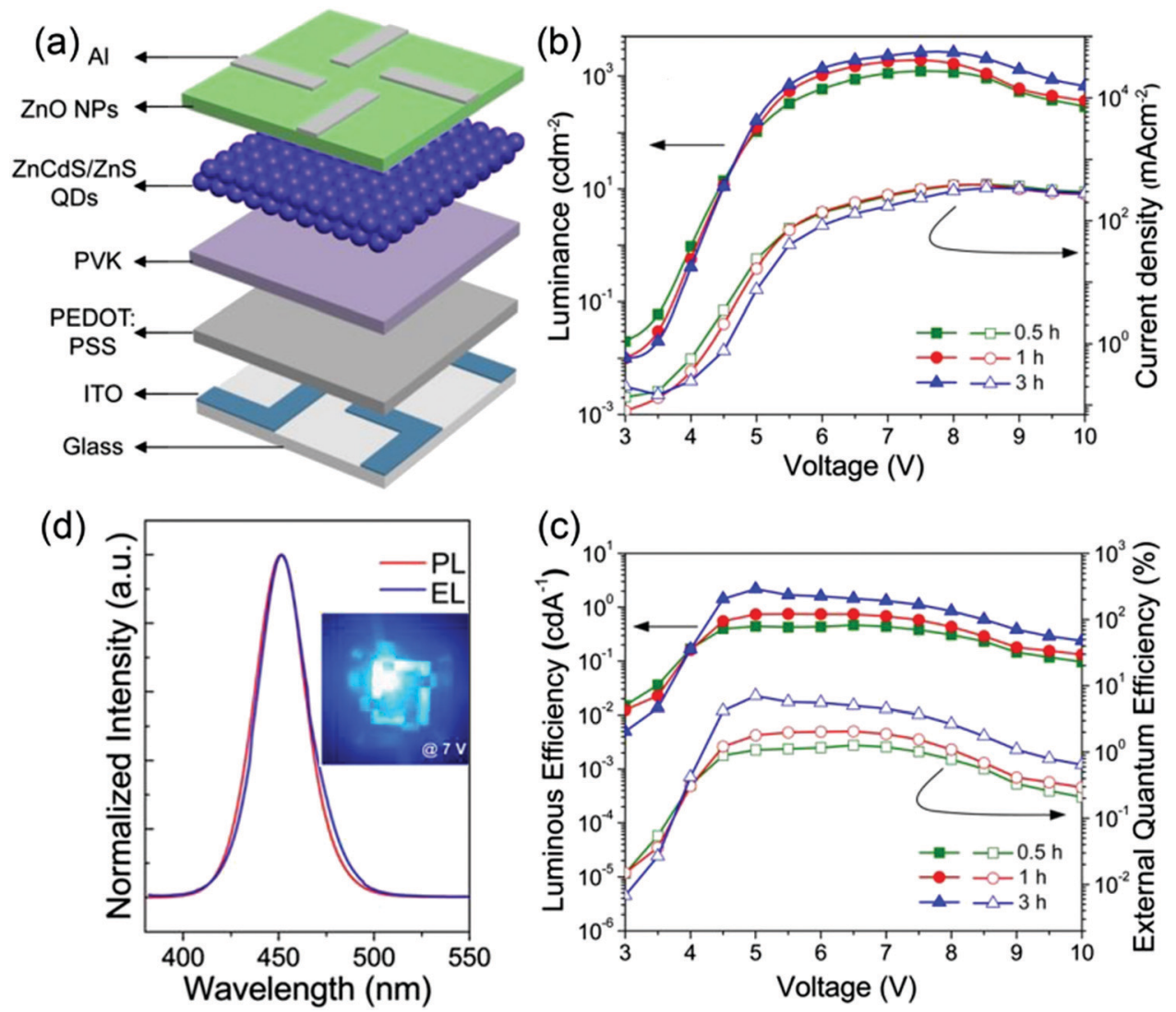

Fig. 2 (a) Schematic illustration of an all-solution-processed blue QD-LED with a multilayered structure, comprising ITO/PEDOT:PSS/PVK/ZnCdS/ZnS QDs/ZnO NPs/Al. Voltage-dependent variations of (b) luminance and current density and (c) current efficiency and $\eta_{\text {EQE }}$ of QD-LED devices based on blue QDs with different shelling times. (d) Spectral comparison of the PL of the QD solution vs. the EL of the QD-LED. The inset shows the EL image at 7 V. Reproduced with permission. ${ }^{35}$ Copyright 2013, American Chemical Society. 
were obtained from 3 h-shelled QD-LEDs (as shown in Fig. 2b and c). In addition, as shown in Fig. 2d, the EL peak position $(452 \mathrm{~nm})$ and FWHM (31 nm) of the $3 \mathrm{~h}$-shelled QD-LEDs almost matched with the PL of the dilute QD solution. However, the only disadvantage in this case was that the turn-on voltage was higher than $4 \mathrm{~V}^{35}$ Hence, to optimize ZnCdS/ZnS QD-based devices, Shen et al. reported a new method, called "nucleation at low temperature/shell growth at high temperature," to synthesize high-quality violet-blue-emitting $\mathrm{Zn}_{x} \mathrm{Cd}_{1-x} \mathrm{~S} / \mathrm{ZnS}$ core/shell QDs. The high growth temperature used therein not only promoted fast shell growth but also promoted the diffusion of $\mathrm{S}$ and $\mathrm{Zn}$ atoms into the $\mathrm{Zn}_{x} \mathrm{Cd}_{1-x} \mathrm{~S}$ core regions. Hence, the surface of $\mathrm{Zn}_{x} \mathrm{Cd}_{1-x} \mathrm{~S}$ cores could be restored and a consecutive change in lattice parameters could be achieved with $\mathrm{Zn}_{x} \mathrm{Cd}_{1-x} \mathrm{~S}$ buffer layers; additionally, stress defects were avoided. Furthermore, by selecting high-quality $\mathrm{Zn}_{x} \mathrm{Cd}_{1-x} \mathrm{~S}$ QDs having the highest PL QY as cores and designing a shell-thickness of $\sim 9.5$ monolayers, the PL QY of the core/shell QDs reached nearly 100\%, which is the highest solution-based value for blue-emitting QDs reported at that time. More importantly, the new well-established protocols proposed by Shen et al. were easy to use on a large scale, and around $37 \mathrm{~g}$ of $\mathrm{Zn}_{x} \mathrm{Cd}_{1-x} \mathrm{~S} / \mathrm{ZnS}$ core/shell QDs could be obtained in one batch reaction. The brightness and efficiency of QD-LEDs with a peak luminance of $4100 \mathrm{~cd} \mathrm{~m}^{-2}$ and a maximum $\eta_{\mathrm{EQE}}$ of $3.8 \%$ were demonstrated by utilizing $\mathrm{Zn}_{x} \mathrm{Cd}_{1-x} \mathrm{~S} / \mathrm{ZnS}$ core/shell QDs as the emitting layer, simultaneously realizing high efficiency and high brightness at that time. ${ }^{36}$ Despite these great advances, the turn-on voltage of more than $3 \mathrm{~V}$ and the operating voltage of more than $5 \mathrm{~V}$ are high, lowering the luminous power efficiency and reducing the operation lifetime of these devices. Additionally, lifetime test results have not been reported for any of the QD-LEDs. Hence, preparing high-quality QDs by carefully controlling their nanostructure and composition is necessary to obtain QD-LEDs with high performance. ZnS is predominantly used as the outer shell to form CdSe/ZnS or CdS/ZnS core/shell QDs. However, a large lattice mismatch between CdS or CdSe and ZnS will cause interfacial strain and reduce the PL QY. The gradual change in composition from the core to the outer shell can effectively relieve the interfacial strain between the core and the shell. In 2015, Qian et al. reported a graded, alloyed intermediate shell $\left(\mathrm{Cd}_{1-x} \mathrm{Zn}_{x} \mathrm{Se}_{1-y} \mathrm{~S}_{y}\right)$ sandwiched between the $\mathrm{Cd}_{1-x} \mathrm{Zn}_{x} \mathrm{~S}$ core and the $\mathrm{Zn}$ - and S-rich outer shell for blue QDs. This device exhibited a maximum $\eta_{\mathrm{EQE}}$ of $10.7 \%$ and a low sub-bandgap turn-on voltage of $2.6 \mathrm{~V}$ through careful optimization of the Cd- and S-rich or Zn- and Se-rich intermediate shell and the thickness of ZnS outer-shell-based QDs. Moreover, the lifetime of the device could reach up to $1000 \mathrm{~h}$, marking the first time when the lifetime of a blue-emitting device was tested. The high device performance can be attributed to (1) the gradual change in composition from the core to the outer shell, which effectively relieved the strain caused by lattice mismatch between the core and the shell, thereby enhancing the QD PL efficiency, and (2) the thinner ZnS outer shell, which enhanced charge injection into QD-LEDs while maintaining high PL QY, generating high efficiency and long lifetime. The outstanding performance of blue QD-LEDs is a critical step toward the commercial realization of QD-LED display technology. ${ }^{20}$
Typically, colloidally synthesized QDs are capped with fatty acids having long hydrocarbon chains that reduce QD conductivity and increase the distance between QDs, affecting the charge injection/transport within the QD layer. Therefore, Cho et al. presented ligand-asymmetric Janus QDs to improve the device performance of QD-LEDs, in which the bottom ligand of QDs in contact with the ZnO ETL could effectively block electrons and the top ligand ensured uniform deposition of organic HTLs with enhanced hole-injection properties. Most importantly, Cho et al. obtained a peak $\eta_{\mathrm{EQE}}$ of $3.23 \%$, evidencing two-fold enhancement as compared with QDs comprising oleic acid (OA) ligands (having a peak $\eta_{\mathrm{EQE}}$ of $\left.1.49 \%\right)$. However, the record $\eta_{\mathrm{EQE}}$ achieved therein was still considerably lower than the calculated maximum device efficiency (est. $\eta_{\mathrm{EQE}}=8 \%$ ) based on the film's PL QY $(40 \%) .{ }^{37}$ Therefore, further investigation of appropriate ligands will improve device performance. Short-chain thioalcohol ligands seem to be a good choice for the following reasons: their strong attachment to the QD surface, the effect of reduction in the space between QDs, the barrier thickness for hole injection into the QDs, and promotion of the valence band energy level of QDs. For instance, in 2015, Shen et al. replaced long-chain ligands (OA) with short ones (OT) to improve the carrier injection/transport properties of QDs. The short-chain ligands (OT) of QDs effectively enhanced hole injection and balanced the electron/hole current in devices. A low turn-on voltage of $2.6 \mathrm{~V}$ and a maximum $\eta_{\mathrm{EQE}}$ of $12.2 \%$ were obtained for these QD-LEDs with a PL peak of 443 $\mathrm{nm} .{ }^{38}$ Based on this work, the peak $\eta_{\mathrm{EQE}}$ of blue QD-LEDs was further improved by optimizing the $\mathrm{ZnS}$ shell thickness of $\mathrm{Zn}_{x} \mathrm{Cd}_{1-x} \mathrm{~S} / \mathrm{ZnS}$ core/shell QDs and choosing appropriate hole transport materials. Although a device that used PVK as the HTL exhibited a peak $\eta_{\mathrm{EQE}}$ of $15.6 \%$, its high turn-on voltage $(5.80 \mathrm{~V})$ resulted in a low maximum luminance of $4500 \mathrm{~cd} \mathrm{~m}^{-2}$ and a low power efficiency of $1.71 \mathrm{~lm} \mathrm{~W} \mathrm{~W}^{-1}$ owing to the low hole mobility of PVK. However, even though the $\eta_{\mathrm{EQE}}$ of a device that used TFB as the HTL was only $11.9 \%$, its maximum luminance reached up to $10400 \mathrm{~cd} \mathrm{~m}^{-2}$ with a turn-on voltage as low as $2.6 \mathrm{~V}$. More importantly, the $\eta_{\mathrm{EQE}}$ of these thick-shell QD-based LEDs was relatively constant over a wide range of luminance values (from 10 to $4000 \mathrm{~cd} \mathrm{~m}^{-2}$ ). In addition, these devices possessed long operating lifetimes with a $T_{70}$ of $47 \mathrm{~h}$ at an initial luminance of $420 \mathrm{~cd} \mathrm{~m}^{-2}$ owing to the ability of the thick QD shells to more effectively confine excitons in the QD cores and minimize the impact on the exciton recombination characteristics resulting from potential variations in the chemical/electronic structures at QD surfaces during device operation such as oxidation/reduction, ligand attachment/detachment, and other defect formations. ${ }^{39}$

Despite the above characteristics, the efficiency and device lifetime of blue QD-LEDs remain lower than those of green and red QD-LEDs, which have a maximum $\eta_{\mathrm{EQE}}$ exceeding $20 \%$ and a device lifetime exceeding $100000 \mathrm{~h}^{20,21}$ Hence, the efficiency and lifetime of deep blue QD-LEDs still need to be enhanced. Although the ZnS thick shell can suppress QD blinking and eliminate photobleaching, it can also create a natural band barrier for charge recombination in QDs. Therefore, based on their reported blue ZnCdS/ZnS core/shell QDs having a PL QY exceeding 90\%, Shen et al. designed ZnCdS core/shell QDs with 
uniform alloyed thick $\mathrm{Cd}_{x} \mathrm{Zn}_{1-x} \mathrm{~S}(x=$ constant $)$ as the intermediate shell and ultrathin $\mathrm{ZnS}$ as the outermost shell. The $\mathrm{Cd}_{x} \mathrm{Zn}_{1-x} \mathrm{~S}$ intermediate shell not only confined excitons within the ZnCdS core to improve the PL QY but also lowered the injection barrier with ultrathin $\mathrm{ZnS}$ as the outermost shell. The resulting $\mathrm{ZnCdS} / \mathrm{Cd}_{x} \mathrm{Zn}_{1-x} \mathrm{~S} / \mathrm{ZnS}$ core/shell QDs exhibited a high PL QY nearing 100\% and a narrow FWHM ( $<18 \mathrm{~nm})$, ensuring high color purity of the related QD-LEDs. As shown in Fig. 3a and b, by changing the thickness of $\mathrm{Cd}_{x} \mathrm{Zn}_{1-x} \mathrm{~S}$ and $\mathrm{ZnS}$ and the ratio of $\mathrm{Zn}$ to $\mathrm{Cd}$ in the intermediate $\mathrm{Cd}_{x} \mathrm{Zn}_{1-x} \mathrm{~S}$ shell, the resulting device exhibited a maximum luminance of $6768 \mathrm{~cd} \mathrm{~m}^{-2}$ and a peak $\eta_{\mathrm{EQE}}$ of $18 \%$ based on $\mathrm{ZnCdS} /$ ${ }_{10 C d} \mathrm{Zn}_{1-x} \mathrm{~S} / 2 \mathrm{ZnS}$ QDs. However, the turn-on voltage was a little high (up to $>6 \mathrm{~V}$ ); hence, the lifetime of the QD-LEDs was only $47.4 \mathrm{~h}$ at an initial luminance of $100 \mathrm{~cd} \mathrm{~m}{ }^{-2}{ }^{40}$ Thus, improvement in the stability of blue QD-LEDs is imminent. In 2018, Lin et al. reported blue nonblinking ( $>98 \%$ “on" time) ZnCdSe/ZnS//ZnS QDs having a PL QY of $92 \%$ by choosing
ZnCdSe alloy QDs as a "good" core and an overcoat of the ZnS shell as the second shell (Fig. 3c). In addition, inserting PMMA between the QD and ZnO ETL interface to impede excessive electron flux produced a maximum luminance of $14100 \mathrm{~cd} \mathrm{~m}^{-2}$ and a peak $\eta_{\mathrm{EQE}}$ of $16.2 \%$, which can be maintained over $12 \%$ in the luminance range of $100-3000 \mathrm{~cd} \mathrm{~m}^{-2}$ (as shown in Fig. 3d). Notably, as observed in Fig. 3e, a device lifetime of $355 \mathrm{~h}$ was obtained at an initial luminance of $100 \mathrm{~cd} \mathrm{~m}^{-2}$. The enhanced device performance might originate from the reduced charge-injection imbalance obtained by inserting the PMMA interlayer, and it may have suppressed all nonradiative exciton quenching processes, such as nonradiative Auger recombination, dissociation of excitons under high fields, and electric-fieldinduced decrease in the PL efficiency of the QDs, due to the interfacial alloyed layer. ${ }^{41}$ However, to date, no blue devices have been reported that can simultaneously possess both high luminance and long lifetime. Recently, Shen et al. replaced the ZnS shell of ZnCdSe/ZnS core/shell QDs with a ZnSe shell,

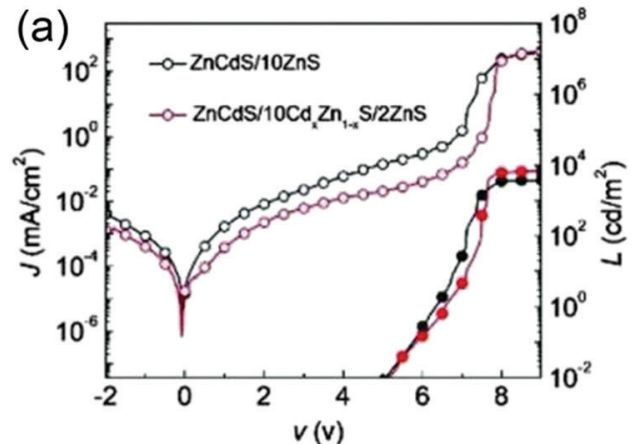

(c)

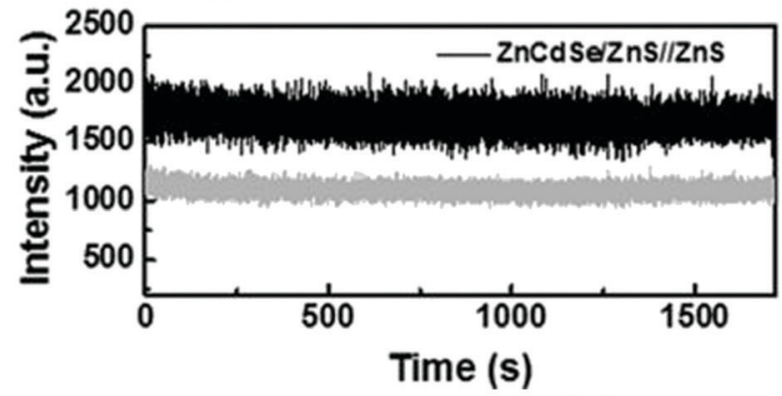

(d)
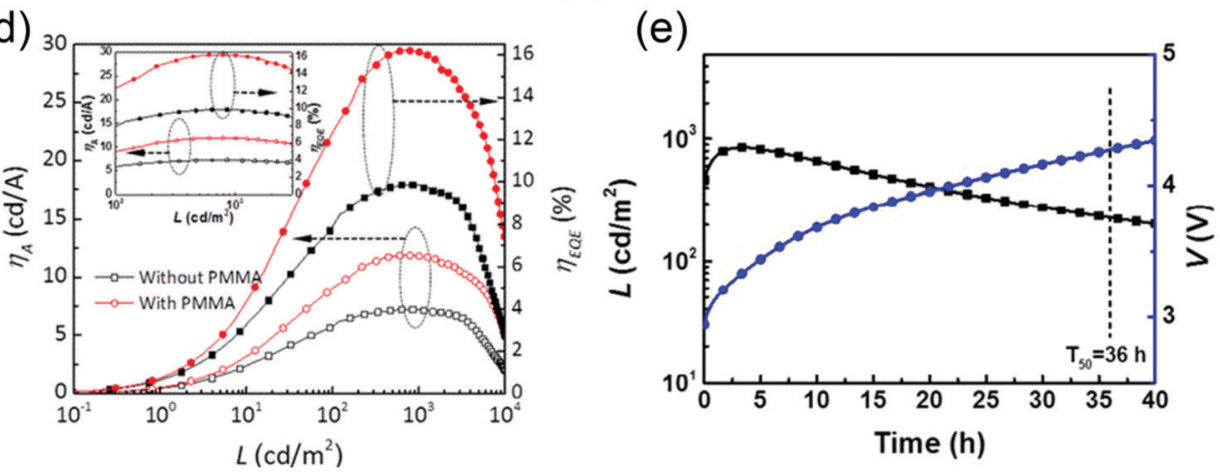

Fig. 3 (a) $J-L-V$ characteristics and (b) current efficiency and $\eta_{\text {EQE }}$ of blue QD-LEDs. Reproduced with permission. ${ }^{40}$ Copyright 2018 , Royal Society of Chemistry. (c) Right: Representative PL intensity time traces (1800 s) of ZnCdSe/ZnS//ZnS core/shell QDs under continuous wave (CW) laser excitation. Left: Photon counting histograms for the fluorescence traces shown on the right. (d) Current efficiency and $\eta_{\text {EQE }}$ obtained using the luminance of QD-LEDs with and without a PMMA layer. The inset shows the corresponding efficiency in the luminance range of $100-3000 \mathrm{~cd} \mathrm{~m}{ }^{-2}$. (e) Operational lifetime characteristics of blue QD-LEDs. Reproduced with permission. ${ }^{41}$ Copyright 2018, American Chemical Society. 
whose smallest valence band offset could match well with the HTL (TFB) compared with other commonly used materials, such as $\mathrm{ZnS}$ and CdS. When incorporating the resulting ZnCdSe/ZnSe core/shell QDs into all-solution QD-LEDs, the $\eta_{\mathrm{EQE}}$ was only 8.05\%; however, the maximum brightness could reach up to $62600 \mathrm{~cd} \mathrm{~m}^{-2}$, which is the highest value reported to date. Moreover, the lifetime of blue QD-LEDs could reach as high as $7000 \mathrm{~h}$ at an initial luminance of $100 \mathrm{~cd} \mathrm{~m}^{-2}$. This device meets the industrial requirements for lighting in terms of both brightness and efficiency (the minimum brightness and $\eta_{\mathrm{EQE}}$ requirements for lighting are $7000 \mathrm{~cd} \mathrm{~m}^{-2}$ and $6 \%$, respectively) as well as device lifetime (for comparison, the average lifetime of incandescent lamps is around $1000 \mathrm{~h}$ ). This work lays a foundation for lighting applications. ${ }^{19}$

\subsection{Optimization of device architecture and interfaces between functional layers}

Despite the significant progress, charge injection/transport imbalance still exists in devices. Apart from the optimization of the QD emitting layer, device architecture optimization also plays a vital role in the enhancement of device performance. In general, the device architecture can be classified into the traditional and inverted structures, in which the QD emitting layer is sandwiched between the ETL and HTL. Electrons and holes are transferred from the cathode and anode to the QD emitting layer through the ETL and HTL, respectively. Therefore, interfacial engineering between the QD emitting layer and CTL together with the judicious choice of electron and hole transport materials will be crucial in balancing the carrier injection and charge transport and enhancing the radiative recombination of excitons in the QD emitting layer. ${ }^{42}$

Owing to the large band-gap and low HOMO level of blue QDs and the relatively shallow HOMO of HTL materials, a large energy barrier exists between the QD emitting layer and HTL, causing difficulty in hole injection from the HTL to QDs. The imbalance between electron and hole injection causes excessive accumulation of electrons in the QD emitting layer, leading to nonradiative Auger recombination outside QDs. Additionally, superfluous electrons without recombination may leak into the HTL, resulting in parasitic emission, thereby affecting the device's color purity. To address this problem, many approaches have been proposed to achieve charge balance, such as amelioration of hole injection or reduction of electron injection. For example, Tan et al. reported a high-performance blue QD-LED by inserting a thin deoxyribonucleic acid (DNA) buffer layer between poly-TPD HTL and $\mathrm{ZnCdS} / \mathrm{ZnS}$ core/shell QD emitting layer. The energy level diagram of the blue QD-LEDs in Fig. 4a demonstrates that the deep-lying HOMO level and the shallow LUMO level of DNA not

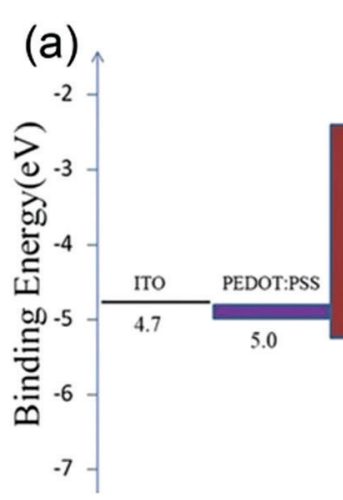

(c)

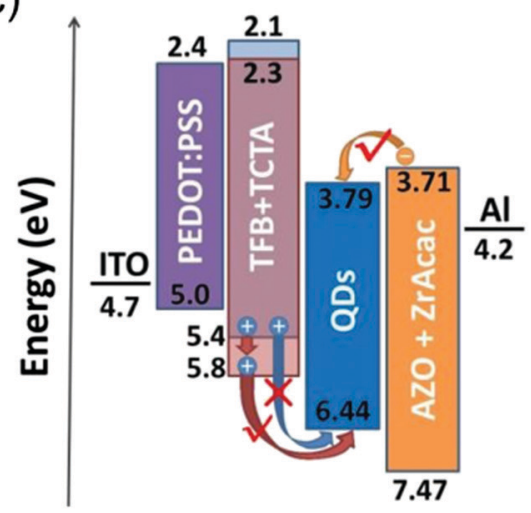

(b)

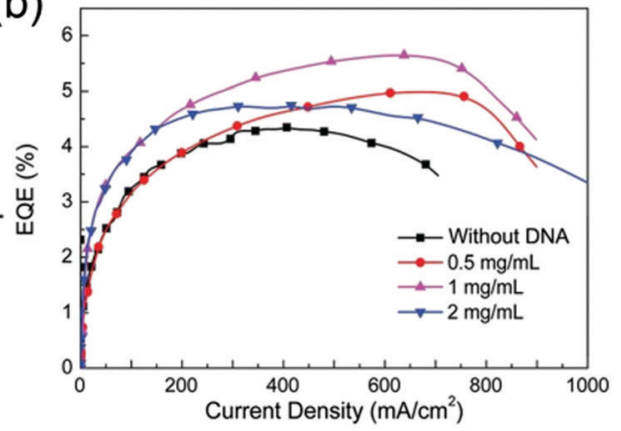

(d)

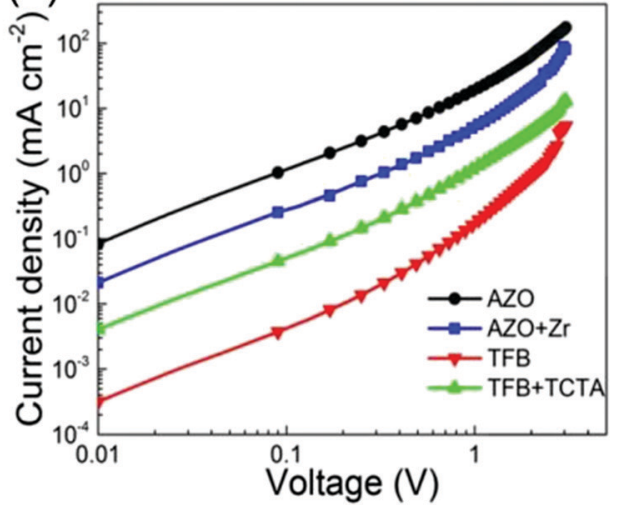

Fig. 4 (a) Energy level diagram of the blue QD-LEDs with a deoxyribose nucleic acid (DNA) modified HTL. (b) $\eta_{\text {EQE }}-$ Current density curves of devices with different DNA concentrations. Reproduced with permission. ${ }^{43}$ Copyright 2018, John Wiley \& Sons, Inc. (c) Energy level diagram of the blue QD-LED with TCTA-doped TFB as well as Al and ZrAcac-doped ZnO NPs as the HTL and ETL, respectively. (d) J-V characteristics of hole-only devices (ITO/ PEDOT:PSS/HTL/QDs/MoO $3 / A l)$ and electron-only devices (ITO/Al/QDs/ETL/Al). Reproduced with permission. ${ }^{45}$ Copyright 2019 , American Chemical Society. 
only effectively enhance hole injection but also confine the injected electrons in the emitting layer, ensuring the charge balance in the QD layer. Consequently, after decorating with DNA, the maximum luminance and $\eta_{\mathrm{EQE}}$ significantly increased from $10218 \mathrm{~cd} \mathrm{~m}^{-2}$ and $4.39 \%$ to $16655 \mathrm{~cd} \mathrm{~m}^{-2}$ and $5.65 \%$, respectively (Fig. 4b). ${ }^{43}$ Apart from introducing additional functional layers, doping HTLs with high-mobility small molecules (such as TCTA or TPD) is another effective strategy to improve the charge mobility and reduce the hole injection barrier of polymer hole-transport materials. The dopant TPD was added to a PVK HTL to form a composite HTL. Because of the reduced injection barrier obtained using stepwise HOMO alignment as well as the reductions of recombination resistance and charge-transfer resistance assessed via electrochemical impedance spectroscopy (EIS) spectra, the maximum $\eta_{\mathrm{EQE}}$ and luminance were significantly increased from $2.37 \%$ and $3997 \mathrm{~cd} \mathrm{~m}^{-2}$ to $8.62 \%$ and $10824 \mathrm{~cd} \mathrm{~m}^{-2}$ when the ratio of TPD and PVK was $1: 1{ }^{44}$ However, the low hole mobility $\left(10^{-6} \mathrm{~cm}^{2} \mathrm{~V}^{-1} \mathrm{~s}^{-1}\right)$ of PVK affected hole transport; hence, TFB (with a high hole mobility of $10^{-2} \mathrm{~cm}^{2} \mathrm{~V}^{-1} \mathrm{~s}^{-1}$ ) is also a sensible choice for high-performance QD-LEDs. Unfortunately, its shallow HOMO produces a large energy offset between $\mathrm{ZnCdS} / \mathrm{ZnS}$ QDs and TFB, rendering it difficult for hole injection. To solve this problem, Tan et al. doped TCTA with low HOMO into TFB as the HTL, guaranteeing both high hole mobility and smooth injection of holes. The corresponding energy level diagram is shown in Fig. 4c. Furthermore, Fig. 4d shows that the hole current density effectively increased in the hole device. To further enhance the balance of charge injection, $\mathrm{Al}$ and ZrAcac doped ZnO NPs were also chosen as the ETL, in which Al doping could adjust the bandgap of ZnO NPs and inhibit blinking in QDs, whereas ZrAcac doping could reduce the electron mobility of the ETL, boosting the charge balance in the QD emitting layer (Fig. 4d). Therefore, the resulting device based on TCTA-doped TFB as well as Al- and ZrAcac-doped ZnO NPs exhibited an exceptionally high luminance of $34874 \mathrm{~cd} \mathrm{~m}^{-2}$ and $\eta_{\mathrm{EQE}}$ of $10.7 \%$, which were 0.87 times and 1.30 times, respectively, higher than those of the control devices. These results prove that doping of CTLs is an effective and simple strategy to regulate charge balance in QD-LEDs. ${ }^{45}$

In addition to the amelioration of hole injection, reduction of electron injection is also an effective method to promote the balance of charge injection, increasing the recombination efficiency in the QD layer. Thus far, various methods have been proposed to optimize electron injection, which could be divided into three categories: (1) inserting a blocking layer between the QDs and ZnO NPs. For instance, inserting a LiF interfacial layer between the ZnO ETL and the QD layer not only facilitated electron injection from $\mathrm{ZnO}$ to QDs through the electron tunneling effect, thereby reducing the accumulation of electrons at the $\mathrm{ZnO} / \mathrm{QD}$ interface, but also suppressed exciton quenching in the QD layer caused by ZnO NPs with a high work function. Consequently, the blue QD-LEDs showed a maximum $\eta_{\mathrm{EQE}}$ of $9.8 \%$, and the operational lifetime of the devices was improved two-fold owing to the more balanced charge injection. ${ }^{46}$ However, the non-conductivity of LiF necessitates that its thickness should be precisely controlled, causing considerable difficulties in industrial production. Compared with
LiF insulating blocking layers, tertbutyldimethylsilyl chloridemodified poly( $p$-phenylene benzobisoxazole) (TBS-PBO) has good conductivity, maintaining current density at a high level. Hence, a peak $\eta_{\mathrm{EQE}}$ of $17.4 \%$ and a maximum luminance of $7992.5 \mathrm{~cd} \mathrm{~m}^{-2}$ were obtained for the device when TBS-PBO was inserted between the ETL and QDs. In particular, a notable luminance of $4635 \mathrm{~cd} \mathrm{~m} \mathrm{~m}^{-2}$ was achieved at the peak $\eta_{\mathrm{EQE}}$ of $17.4 \%$, which was the highest luminance reported for blue QD-LEDs. This work offers a potential approach to achieve blue QD-LEDs with high luminance. ${ }^{47}$ (2) Doping metallic ions to tune the conductivity and optical bandgap of ZnO NPs. According to Sun's report, through doping $\mathrm{Al}$ ions, the work function and conduction band edge of $\mathrm{ZnO}$ were raised by generating additional free electrons in the conduction band, effectively suppressing charge transfer at the QD/ZnO interface. Consequently, the blue QD-LEDs exhibited a maximum $\eta_{\mathrm{EQE}}$ of $4.0 \%$ and a decreased turn-on voltage from 4.1 to $3.8 \mathrm{~V}^{48}$ (3) Modifying the ZnO ETLs to tune the trap state density in ZnO NPs. For instance, post-annealing the $\mathrm{ZnO}$ film is the most typical treatment method that remarkably impacts device performance. Although the bandgap of the $\mathrm{ZnO}$ film is not influenced by annealing temperature, the gap state density decreases with increasing annealing temperature, indicating the drop in electron mobility in ZnO NPs. Additionally, they introduced $\mathrm{Al}: \mathrm{Al}_{2} \mathrm{O}_{3}$ as the cathode to reduce the energy barrier between the QDs and the cathode, resulting in improvement of exciton confinement. Thus, with the good cooperation of the postannealed $\mathrm{ZnO}$ ETL and the $\mathrm{Al}: \mathrm{Al}_{2} \mathrm{O}_{3}$ cathode, a high brightness of $27753 \mathrm{~cd} \mathrm{~m}^{-2}$ and $\eta_{\mathrm{EQE}}$ of $8.92 \%$ were obtained when the $\mathrm{ZnO}$ annealing temperature was $80{ }^{\circ} \mathrm{C}$ (Fig. 5a and b). ${ }^{49}$ The electron mobility of the ZnO NPs can also be adjusted by controlling their particle size. Wang et al. synthesized two types of ZnO NPs A $(d=3.626 \mathrm{~nm})$ and B $(d=3.888 \mathrm{~nm})$ and investigated the effect of different-sized $\mathrm{ZnO}$ NPs on device performance. That study showed that small-size ZnO NPs A had a low surface roughness and more trap states than ZnO NPs B; hence, the electron-only device based on ZnO NPs B would have a larger leakage current than that based on ZnO NPs A. Consequently, the blue QD-LEDs based on ZnO NPs A and CdSe/ ZnS QDs with a PL QY of $87 \%$ exhibited an exceptionally high $\eta_{\mathrm{EQE}}$ of $19.8 \%$ at a current density of $5.53 \mathrm{~mA} \mathrm{~cm}^{-2}$, and the device efficiency is the highest value among previous reports (Fig. 5c). The QD-LEDs had pure blue light with a narrow FWHM of $20 \mathrm{~nm}$ corresponding to CIE 1931 color coordinates of $(0.136,0.078)$, which are very close to the NTSC 1953 standard of $(0.14,0.08)$ (Fig. $5 d$ ). Its color saturation property thus makes this QD-LED an ideal blue light source for display applications. ${ }^{33}$

In the conventional QD-LED structure, an orthogonal solvent must be chosen to prevent physical damage during solution-based deposition. Now, the inverted device architecture is adopted to solve the problem of solvent selection restrictions. In addition, QD-LEDs with an inverted structure are highly advantageous for display applications because the bottom transparent cathode can be directly connected to n-channel metal-oxide or amorphous silicon-based thin-film transistor (TFT) backplanes. According to Kwak's report, the inverted blue device obtained by adopting the solution-processed ZnO NP film as the ETL and 

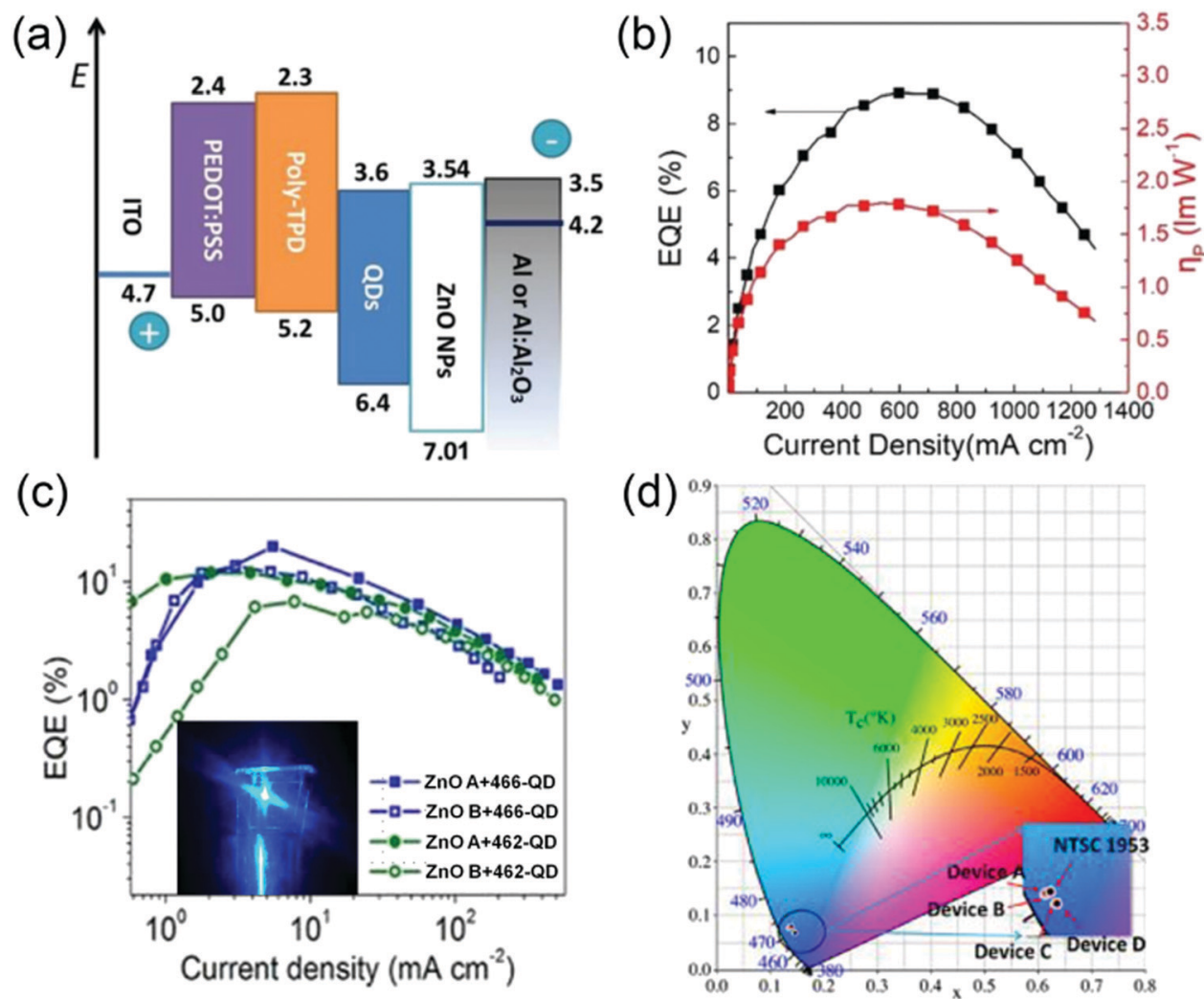

Fig. 5 (a) Energy level diagram of the as-prepared QD-LEDs. (b) $\eta_{\mathrm{EQE}}-$ power density curves of the best device with $\mathrm{ZnO}(\mathrm{a}) / \mathrm{Al}: \mathrm{Al}_{2} \mathrm{O}_{3}$. $\mathrm{Reproduced}$ with permission. ${ }^{49}$ Copyright 2019, John Wiley \& Sons, Inc. (c) $\eta_{\text {EQE }}$-current density curves of devices with different ZnO and QDs. Inset: Image of the device with small-size ZnO NPs A and high PL QY (87\%) QDs at a driving voltage of 7 V. (d) CIE properties. Reproduced with permission. ${ }^{33}$ Copyright 2017 , American Chemical Society.

vacuum evaporated CBP and $\mathrm{MoO}_{3}$ as the $\mathrm{HTL} /$ hole injection layer (HIL) demonstrated a high efficiency of $1.7 \%$ and a low turn-on voltage of $3.0 \mathrm{~V}$, which directly corresponded to the optical bandgap of blue $\mathrm{Cd}_{1-x} \mathrm{Zn}_{x} \mathrm{~S} @ Z \mathrm{ZnS}$ QDs. This significantly improved performance might result from direct and efficient carrier recombination within QD layers caused by the facilitated charge carrier injection into QDs from ZnO or CBP. ${ }^{50}$ Together with a deepening understanding of the physical mechanism of devices, the optimization of device structure and engineering of QD emitting material, the device performance of QD-LEDs has been further improved. For example, Zhong et al. presented an inverted blue QD-LED by inserting an ultra-thin PEI between the ITO and ZnO layers to decrease oxygen vacancy defects of ZnO and electron accumulation at the interface of the $\mathrm{ZnO}$ and QDs. Consequently, the blue QD-LEDs exhibited an increased peak $\eta_{\mathrm{EQE}}$ from $3.5 \%$ to $5.5 \% .^{51}$ However, vacuum evaporation is a high-cost technology that is unsuitable for large-area applications. Recently, all-solution-processed inverted blue QD-LEDs have been developed. The first one was reported by Castan $e t$ al., who used polyoxyethylene tridecyl ether (PTE) to facilitate the deposition of aqueous PEDOT:PSS on a hydrophobic polymer hole transport material. However, the current efficiency of the blue QD-LEDs was merely $0.06 \mathrm{~cd} \mathrm{~A}^{-1}$ owing to the effect of PTE on decreasing current flow through the device. ${ }^{52}$ To further resolve the solvent erosion of the HTL on the QD emitting layer, Liu et al. adopted the polar solvent 1,4-dioxane as the solvent of the PVK HTL and phosphomolybdic acid hydrate (PMAH) dissolved in isopropanol as the HIL. The resulting blue QD-LEDs exhibited a maximum luminance of $1280 \mathrm{~cd} \mathrm{~m}^{-2}$ and a peak $\eta_{\mathrm{EQE}}$ of $4.69 \%$. To further enhance the EL performance of the devices, a double-layer HTL, $\mathrm{PVK} / \mathrm{TFB}$, was developed to provide stepwise hole injection, wherein the second HTL layer TFB dissolved in $p$-xylene prevented physical damage to the PVK layer. Therefore, a high $\eta_{\mathrm{EQE}}$ of $5.99 \%$ and a maximum brightness of $4140 \mathrm{~cd} \mathrm{~m}^{-2}$ were obtained at an EL peak of $454 \mathrm{~nm}$, and the device efficiency is the highest value among all inverted QD-LEDs, including thermally evaporated devices. ${ }^{53}$ However, $\eta_{\mathrm{EQE}}>20 \%$ has been routinely reported in red and green QD-LEDs, whereas it is still rarely reported in blue QD-LEDs. A promising approach to simultaneously achieve high efficiency and long operational lifetime is to employ a tandem structure, which has been widely adopted in OLEDs. In the case of blue QD-LEDs, an efficient tandem QD-LED with an $\eta_{\mathrm{EQE}}$ exceeding $21 \%$ was reported using an ICL with the structure of $\mathrm{ZnMgO} / \mathrm{Al} / \mathrm{dipyrazino}\left[2,3-f: 2^{\prime}, 3^{\prime}\right.$ h]quinoxaline-2,3,6,7,10,11-hexacarbonitrile (HATCN)/ $\mathrm{MoO}_{3}$. The proposed ICL exhibits high transparency, efficient charge generation/injection capability, and high robustness to resist solvent damage during the deposition of the upper functional layers, and thus the highly bright blue QD-LEDs demonstrated a record $\eta_{\mathrm{EQE}}$ and current efficiency of $21.4 \%$ and $17.9 \mathrm{~cd} \mathrm{~A}^{-1}$, respectively, 

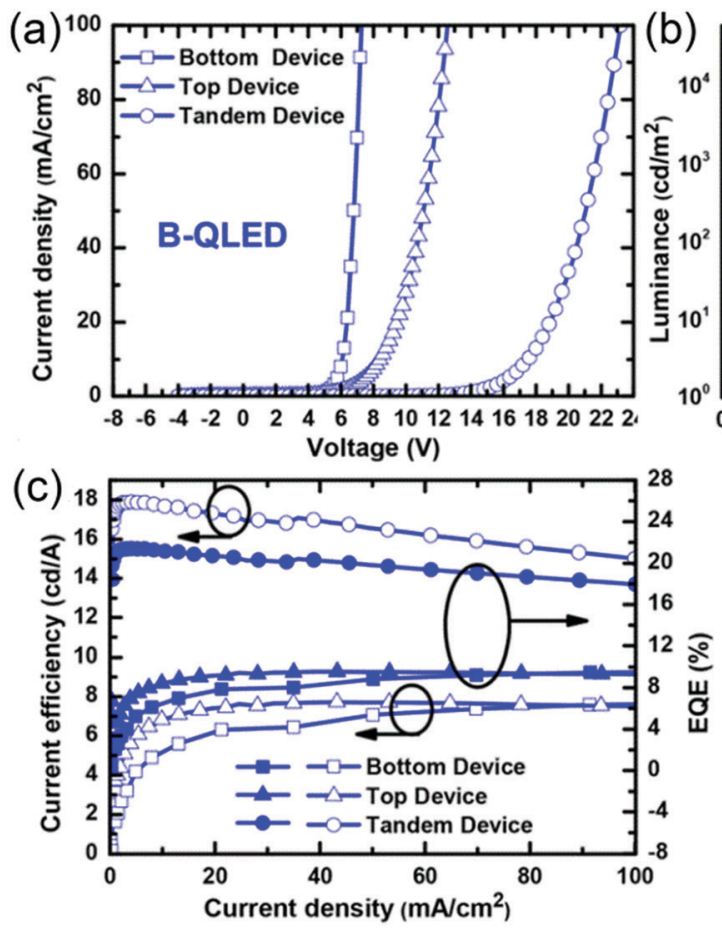

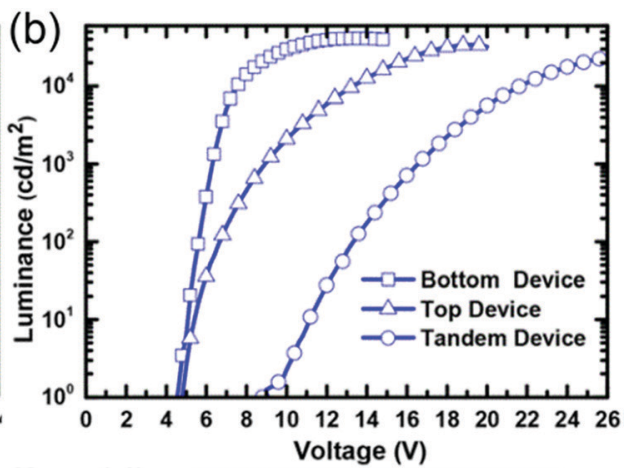

(d)

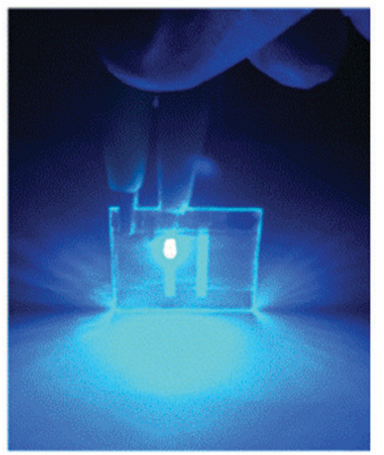

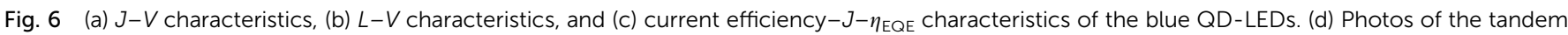

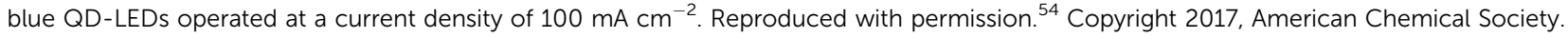

and a high luminance of $26800 \mathrm{~cd} \mathrm{~m} \mathrm{~m}^{-2}$ (Fig. 6); these results render blue QD-LEDs ideal candidates to bring QD-LEDs into the next generation of full-color displays and solid-state lighting markets. ${ }^{54}$

All the results presented above are based on ITO-patterned glass substrates, whose rigidity limits the applications in foldable displays. Therefore, flexible photoelectric devices are necessary for next-generation displays. In 2017, Ji et al. reported highperformance red, green, and blue three-primary-color QD-LEDs based on the flexible PET (poly(ethylene-terephthalate))/ITO/Ag/ ITO (IAI) transparent cathode for the first time. The IAI film on PET possesses similar transmittance to that of a pure ITO film, and ultrastability after bending 2000 times with a bending radius of $5 \mathrm{~mm}$. When applied to QD-LEDs with a structure of PET/IAI/ZnO/ QDs/mCP/ $/ \mathrm{MoO}_{x} / \mathrm{Al}$, blue QD-LEDs display good EL performance with a maximum current efficiency (luminance) of $16.1 \mathrm{~cd} \mathrm{~A}^{-1}$ $\left(18127 \mathrm{~cd} \mathrm{~m}^{-2}\right)$. This work unlocked a new opportunity for flexible QD-LEDs in terms of application to displays and lightings. ${ }^{55}$

\subsection{The aging mechanism of blue QD-LEDs}

In the experiment, we find that the performance of QD-LEDs is significantly enhanced after shelf-aging for several days. This phenomenon can be termed as positive aging. Acharya et al. considered that positive aging was caused by the encapsulation resin, which slowly reacted with the $\mathrm{ZnO}$ surface and ambient carbon dioxide, thus forming zinc carbonate that helped to reduce $\mathrm{ZnO} \mathrm{NP}$ defect densities and modified the $\mathrm{ZnO} / \mathrm{QDs}$ or $\mathrm{ZnO} / \mathrm{Al}$ interface. ${ }^{16}$ However, Chen et al. determined that the encapsulation resin did not play a major role in positive aging. To find out the exact origin of positive aging, the effects of cathode materials, ETL materials, and QDs on the aging behavior were systematically investigated by Chen et al. To rule out the effect of the cathode, a device without an $\mathrm{Al}$ cathode was fabricated initially, and after five days, the Al cathode was deposited and the device was characterized immediately. As shown in Fig. 7a, the device with the Al cathode deposited after five days did not show positive aging and exhibited a decreased $\eta_{\mathrm{EQE}}$ from $5.9 \%$ to $3.7 \%$, indicating that positive aging remained unaffected by QDs and ZnMgO ETL. However, the device with the $\mathrm{Al}$ cathode deposited initially exhibited an enhanced $\eta_{\mathrm{EQE}}$ from $5.9 \%$ to nearly $9.0 \%$ after five days, which might have been induced by the $\mathrm{Al} / \mathrm{ZnMgO}$ interface. To precisely probe the $\mathrm{Al} / \mathrm{ZnMgO}$ interface, the $\mathrm{Al}$ was removed via sputter etching and the surface was monitored via X-ray photoelectron spectroscopy (XPS) (Fig. 7b and c). The intensity of O-2, which was related to the content of oxygen vacancies and oxygen bonds in the hydroxide, was higher than that of the fresh sample after five days of aging, whereas the $\mathrm{Al}-2$, corresponding to amorphous $\mathrm{AlO}_{x}$, was also enhanced after five days. This can be attributed to the fact that the oxygen outdiffused from the $\mathrm{ZnMgO}$ layer and then reacted with $\mathrm{Al}$, thus accumulating oxygen vacancies at the $\mathrm{Al} / \mathrm{ZnMgO}$ interface and forming $\mathrm{AlO}_{x}$. The oxygen vacancies at the $\mathrm{Al} / \mathrm{ZnMgO}$ interface may have increased the electron concentration of $\mathrm{ZnMgO}$ and the $\mathrm{AlO}_{x}$ interlayer could have effectively suppressed the quenching of excitons through the metal electrode, enhancing the performance of blue QD-LEDs. ${ }^{17}$ Furthermore, positive aging can also be observed under accelerated processes, such as stressing by a continuous constant current and post metallization annealing (Fig. $7 \mathrm{~d}$ and e). ${ }^{17,18}$ 

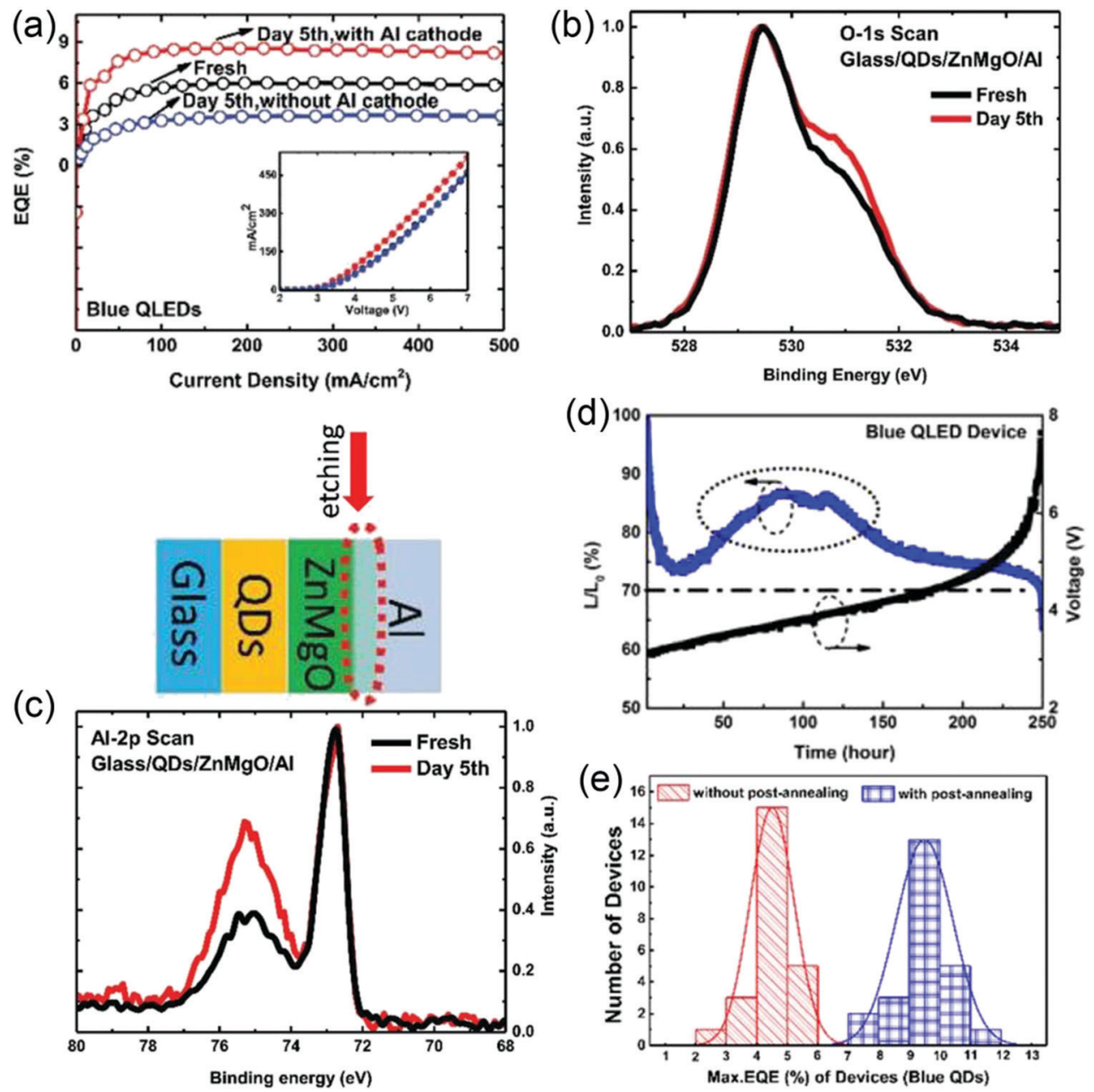

Fig. 7 (a) $\eta_{\mathrm{EQE}}$-current density characteristics of blue QD-LEDs with and without the Al cathode placed initially. X-ray photoelectron spectroscopy (XPS) spectra of the (b) O 1s scan and (c) Al 2p scan at the Al/ZnMgO interface. (d) Lifetime of blue QD-LEDs with a conventional structure. Reproduced with permission. ${ }^{17}$ Copyright 2018, John Wiley \& Sons, Inc. (e) Quantity statistics of the maximum $\eta_{\mathrm{EQE}}$ of blue QD-LEDs without and with post annealing. Reproduced with permission. ${ }^{18}$ Copyright 2018, American Chemical Society.

\section{Blue perovskite LEDs}

Owing to their bright tunable emissions and extremely narrow line widths, lead halide perovskite QDs have also attracted interest for potential optoelectronic device applications. In recent years, impulsive progress has been made in lead halide PeLEDs, and their maximum $\eta_{\mathrm{EOE}}$ has increased from $0.76 \%$ to more than $20 \%{ }^{22,23,56-64}$ In 2014, the first PeLEDs were reported by Tan et al., and the resultant $\eta_{\mathrm{EQE}}$ values for nearinfrared, red, and green emission at room temperature were $0.76 \%, 0.1 \%$, and $0.018 \%$, respectively. This demonstration of effective perovskite EL offers scope for developing this unique class of materials into efficient and color-tunable light emitters for low-cost display, lighting, and optical communication applications. ${ }^{56}$ Ever since, the efficiency and luminance of PeLEDs have been drastically improved through composition and morphology engineering, surface modification, and device engineering. Thus far, near-infrared, red, and green PeLEDs have possessed high $\eta_{\mathrm{EQE}}$ values of up to $20.7 \%,{ }^{64} 21.3 \%,{ }^{23}$ and $20.3 \%,{ }^{22}$ respectively. However, the development of blue PeLEDs is relatively slow. In the following sections, we will review the recent progress of blue PeLEDs based on the types of perovskite materials, including 2D/quasi 2D perovskites included in organic-inorganic halide perovskites and all inorganic perovskites; additionally, Table 2 summarizes detailed recent advances in blue PeLEDs.

\subsection{Organic-inorganic PeLEDs}

The first blue PeLEDs based on organic-inorganic halide perovskites were reported by Kumawat $e t$ al. in 2015, who used PEDOT: PSS, $\mathrm{MAPBBr}_{1.08} \mathrm{Cl}_{1.92}$, and PCBM as the HIL, emitting layer, and ETL, respectively. However, the corresponding device exhibited a poor brightness of $2 \mathrm{~cd} \mathrm{~m}^{-2}$ and a peak $\eta_{\mathrm{EQE}}$ of $0.0001 \%$ at room temperature owing to the pinholes in the perovskite film and emission quenching caused by PCBM. ${ }^{65}$ Therefore, to reduce pinholes in the perovskite film, Kim et al. reported a triple-cation strategy that incorporated Cs cations into a mixed cation perovskite: $\mathrm{MA}_{0.17} \mathrm{FA}_{0.83} \mathrm{~Pb}\left(\mathrm{Br}_{x} \mathrm{I}_{1-x}\right)_{3}$. In addition, using the two-step spin-coating method to form highly dense and pinhole-free perovskite films and by choosing an appropriate 
Table 2 Recent advances in blue PeLEDs

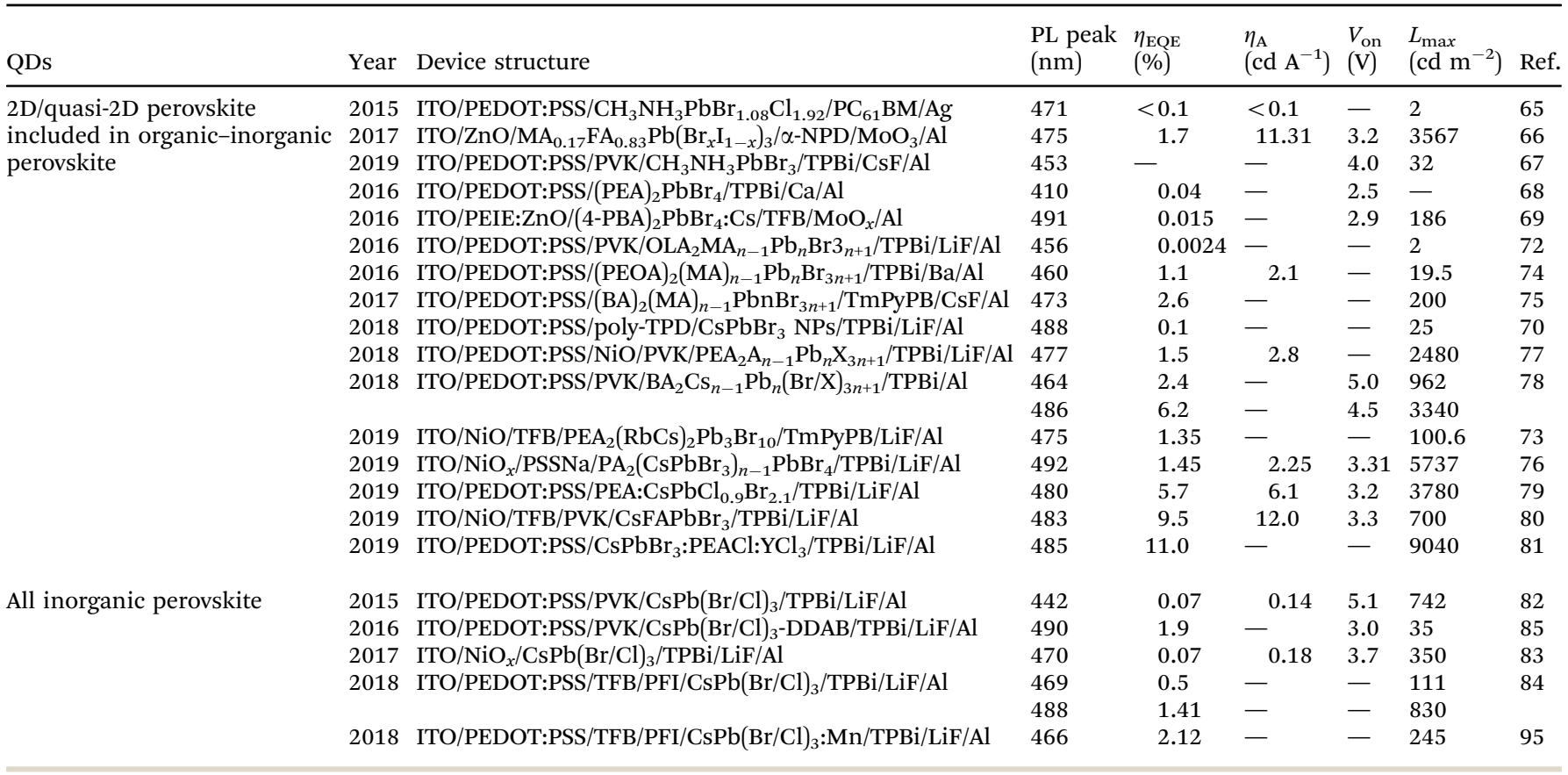

ETL of ZnO NPs and an HTL of $\alpha$-NPD, the resulting device delivered a high brightness of $3567 \mathrm{~cd} \mathrm{~m}^{-2}$, a peak $\eta_{\mathrm{EQE}}$ of $1.7 \%$, and a low turn-on voltage of $3.2 \mathrm{~V}$, which directly corresponded to the optical bandgap of the perovskite (Fig. 8a). The device also showed remarkably stable EL spectra at different bias voltages (Fig. 8b). However, the long-term stability of these devices under operating conditions remains a challenge. Kim et al. evaluated the operating lifetime of the device at room temperature and a constant current density for an initial luminance of $374.5 \mathrm{~cd} \mathrm{~m}^{-2}$, the result of which demonstrated that during the first $25 \mathrm{~min}$, the luminance increased to $948.5 \mathrm{~cd} \mathrm{~m}^{-2}$ and then rapidly decreased to $88.5 \mathrm{~cd} \mathrm{~m}^{-2}$ after $150 \mathrm{~min}^{66}$ In addition, to further promote their potential commercialization applications, efficient mass production of colloidal perovskite QDS has become an important research topic. In 2018, Zhong et al. reported a modified emulsion synthesis by introducing the phase transfer strategy to fabricate gram-scale $\mathrm{CH}_{3} \mathrm{NH}_{3} \mathrm{PbBr}_{3}$, which achieved effective extraction of newly formed perovskite QDs into non-polar solvent and avoided the degradation of perovskite QDs to a large extent. The as-fabricated $\mathrm{CH}_{3} \mathrm{NH}_{3} \mathrm{PbBr}_{3}$ QDs exhibited a high PL QY of more than $70 \%$ and an emission peak of $453 \mathrm{~nm}$ with a FWHM of only $14 \mathrm{~nm}$. However, the peak luminance of the related device was only $32 \mathrm{~cd} \mathrm{~m}^{-2}$ and the FWHM of the EL spectrum was broadened to $16 \mathrm{~nm} .{ }^{67}$ Therefore, investigating more stable materials, such as $2 \mathrm{D} /$ quasi-2D organic-inorganic perovskite or all-inorganic perovskite materials, is necessary.

Although efficient blue electroluminescent three-dimensional (3D) PeLEDs have been successfully fabricated, the highest reported $\eta_{\mathrm{EQE}}$ for blue PeLEDs is still less than $5 \%$. A convenient approach to create blue-emission perovskite films is transitioning from $3 \mathrm{D}$ lead halide perovskites into $2 \mathrm{D}$ or quasi-2D structures, which will introduce quantum confinement effects in these materials, and therefore, the PL peak can be easily tuned into blue or even violet wavelengths. For example, Liang et al. first

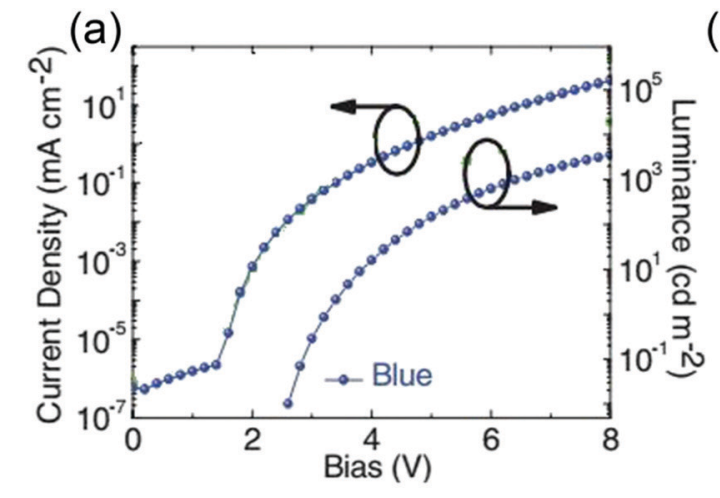

(b)

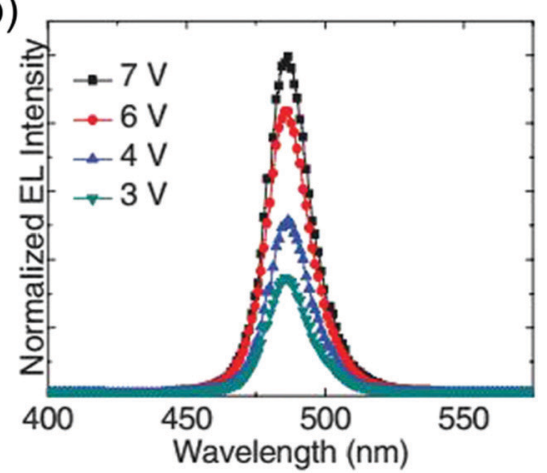

Fig. 8 (a) $J-L-V$ characteristics for blue PeLEDs based on Cs-doped $M A_{0.17} F A_{0.83} P b\left(B r_{x} l_{1-x}\right)_{3}$. (b) EL spectra of the blue-colored PLEDs operating at various voltages. Reproduced with permission. ${ }^{66}$ Copyright 2017, John Wiley \& Sons, Inc. 
reported violet PeLEDs based on $2 \mathrm{D}$ perovskites using (PEA) ${ }_{2} \mathrm{PbBr}_{4}$ (PEA = phenylethylammonium) NPs as the emitting layer and delivered a maximum $\eta_{\mathrm{EQE}}$ of $0.04 \%$ at room temperature. ${ }^{68}$ By changing the organic spacer layer and mixing inorganic (Cs) cations, the related sky-blue PeLED based on ITO/ZnO/PEIE/ (4-PBA) ${ }_{2} \mathrm{PbBr}_{4}: \mathrm{Cs} / \mathrm{TFB} / \mathrm{MoO}_{3} / \mathrm{Al}$ exhibited a peak luminance of $186 \mathrm{~cd} \mathrm{~m}^{-2}$ and a maximum $\eta_{\mathrm{EQE}}$ of $0.015 \%$ at an EL peak of $491 \mathrm{~nm} .{ }^{69}$ Moreover, Yang et al. demonstrated a simple one-pot colloidal approach to synthesize ultrathin $\mathrm{CsPbBr}_{3}$ nanoplates (NPs) of tunable edge length and thickness. The blue PeLED of ITO/PEDOT:PSS/poly-TPD/CsPbBr ${ }_{3}$ NPs/TPBi/LiF/Al exhibited a luminance of $25 \mathrm{~cd} \mathrm{~m} \mathrm{~m}^{-2}$ and an $\eta_{\mathrm{EQE}}$ of $0.1 \% .^{70}$ However, 2D perovskites have limited PL QYs owing to their strong exciton-phonon coupling, causing difficulties in enhancing device performance.

The combination of $2 \mathrm{D}$ and $3 \mathrm{D}$ perovskites named quasi-2D perovskites with the formula of $\left(\mathrm{RNH}_{3}\right)_{2} \mathrm{~A}_{n-1} \mathrm{~B}_{n} \mathrm{X}_{3 n+1}$ (where $n$ is the number of stacking perovskite octahedra and the bandgap can be tuned by changing the $n$ values) was reported as a useful strategy to grant these materials with both compact pinholefree morphology and excellent optoelectronic properties such as high PL QYs and good charge carrier balance. ${ }^{71}$ In 2016, Kumar et al. prepared colloidal, quantum-confined 2D perovskites $\mathrm{OLA}_{2} \mathrm{MA}_{n-1} \mathrm{~Pb}_{n} \mathrm{Br}_{3 n+1}$ using low- $k$ organic host compounds to boost the exciton binding energy; the PL QYs of these perovskites could reach up to $40-90 \%$. By tuning the $n$ value from 1 to 10 , they achieved tunable EL with $\lambda=432,456,492$, and $520 \mathrm{~nm}$ at room temperature, and a blue PeLED with an emission peak at $456 \mathrm{~nm}$ exhibited an $\eta_{\mathrm{EQE}}$ of $0.0024 \%$ and a brightness of $2 \mathrm{~cd} \mathrm{~m}^{-2}$. $^{72}$ The poor device performance may be due to the inefficient internal energy transfer and Ostwald ripening because of the extremely low $n(n \leq 2)$ quasi-2D perovskites. So, Jiang et al. fabricated a Rb-Cs alloyed, $n_{\mathrm{Rb} 0.6}=3$ quasi-2D perovskite by tuning the 'A-site' cation composition, and demonstrated its great potential for further pure-blue LED application. The fabricated quasi-2D perovskite showed a prominent film PL QY of around $82 \%$ at $476 \mathrm{~nm}$, and there was no PL spectral shift or broadening in these materials even after long-time thermal annealing. Eventually, the related PeLEDs exhibited a peak $\eta_{\mathrm{EQE}}$ of $1.35 \%$ and a lifetime of $14.5 \mathrm{~min}$ at the maximum $\eta_{\mathrm{EQE}}$ point. However, the maximum brightness was only $100.6 \mathrm{~cd} \mathrm{~m}^{-2}$, which may be attributed to the poor perovskite film. ${ }^{73}$ Chen et al. also found that device performance strongly depends on the perovskite film structure. They added a new organic material 2-phenoxyethylamine (POEA) into the $\mathrm{CH}_{3} \mathrm{NH}_{3} \mathrm{PbBr}_{3}$ precursor solution, causing the transition from bulk film to layered structure through $\pi-\pi$ stacking. This transition drastically changed the PL and EL of $\mathrm{CH}_{3} \mathrm{NH}_{3} \mathrm{PbBr}_{3}$ with emission color being shifted from green to blue, and the associated performance of PeLEDs extraordinarily improved under optimized conditions, reaching a brightness of $19.5 \mathrm{~cd} \mathrm{~m}^{-2}$ and an $\eta_{\mathrm{EQE}}$ of $1.1 \%{ }^{74}$ In addition, the introduction of large-group ammonium halides is another method to obtain layered perovskites. Wang et al. found that the excess ethylammonium bromide (EABr) caused the phase transition from bulk to layered perovskite, accompanied by a blue shift of $40 \mathrm{~nm}$. The associated PeLEDs with the EL located at $473 \mathrm{~nm}$ realized a peak $\eta_{\mathrm{EQE}}$ of $2.6 \%$ and a power efficiency of
$1 \mathrm{~lm} \mathrm{~W}^{-1}$ at $100 \mathrm{~cd} \mathrm{~m}^{-2}$, exhibiting substantial improvement over those of previously reported analogous devices. ${ }^{75}$ In addition to the improvements of perovskites, adopting a suitable carrier (hole and electron) transport/injection layer to reduce the carrier injection barrier is another way to improve the performance of blue PeLEDs. Although $\mathrm{NiO}_{x}$ was widely used as an efficient HTL in perovskite photovoltaics, it has been limitedly studied in blue PeLEDs because of its quenching effect on the perovskite emitting layer. Therefore, Ren et al. demonstrated an efficient hole transport bi-layer structure composed of PSSNa and $\mathrm{NiO}_{x}$, which can not only inhibit the nonradiative decay between $\mathrm{NiO}_{x}$ and perovskite films by reducing $\mathrm{NiO}_{x}$ surface defects, but can also improve quasi-2D perovskite thin film quality by minimizing its pin-holes and reducing the film roughness. Eventually, the blue PeLED was achieved with a very low turn-on voltage of $3.31 \mathrm{~V}$ accompanied with an $\eta_{\mathrm{EQE}}$ of $1.45 \%$ and a remarkable luminance of $5737 \mathrm{~cd} \mathrm{~m}{ }^{-2}{ }^{76}$ However, the abovementioned blue PeLEDs usually exhibited poor color stability under LED operation because of the presence of multiple emission peaks arising from mixed phases. Therefore, Sargent et al. slowed the formation of the pure $n=1$ phase to enhance the purity and monodispersity of $n=2,3$, and 4 phases by replacing long ligands (PEA) with shorter ones (iso-propylammonium, IPA). The as-synthesized perovskite films exhibited a single emission peak $(474 \mathrm{~nm})$ with a record PL QY of $88 \%$, and the resulting PeLEDs exhibited a maximum luminance of $2480 \mathrm{~cd} \mathrm{~m}^{-2}$ and a peak $\eta_{\mathrm{EQE}}$ of $1.5 \%$. Particularly, the EL peak of $490 \mathrm{~nm}$ and the FWHM of $28 \mathrm{~nm}$ remained unchanged when the applied voltage increased from 5 to $10 \mathrm{~V} .^{77}$ Besides, Vashishtha et al. adopted butylammonium as a separating ligand to create $\mathrm{BA}_{2} \mathrm{Cs}_{n-1} \mathrm{~Pb}_{n}(\mathrm{Br} / \mathrm{Cl})_{3 n+1} 2 \mathrm{D} / 3 \mathrm{D}$ mixed halide thin films, in which changing the butylammonium concentration could optimize the number of $\mathrm{CsPbBr}_{3}$ monolayers. When they were incorporated into PeLEDs with a structure of ITO/PEDOT:PSS/PVK/ $\mathrm{BA}_{2} \mathrm{Cs}_{n-1} \mathrm{~Pb}_{n}(\mathrm{Br} / \mathrm{X})_{3 n+1} / \mathrm{TPBi} / \mathrm{Al}$, a record efficiency of $6.2 \%$ at $487 \mathrm{~nm}$ for blue-emitting PeLEDs was obtained, and the peak luminance could reach up to $3340 \mathrm{~cd} \mathrm{~m}^{-2}$ (Fig. 9a and b). ${ }^{78}$ However, the relatively poor thermal stability is detrimental to device stability, which requires a high working voltage and hence a strong electric field for operation. Therefore, a much more stable perovskite system is required for blue PeLEDs. Among the perovskite family, an all-inorganic perovskite system possesses much better structural and thermal stability than an organic-inorganic hybrid system. Hence, a more stable blue PeLED can be obtained by forming a quasi-2D perovskite from a green-emissive all-inorganic 3D perovskite of $\mathrm{CsPbBr}_{3}$. In 2019, Li et al. first synthesized $\mathrm{CsPbCl}_{0.9} \mathrm{Br}_{2.1}$ with emission at $484 \mathrm{~nm}$ through composition engineering by incorporating $\mathrm{Cl}$ into the $\mathrm{CsPbBr}_{3}$ lattice, and then introduced phenylethylammonium bromide (PEABr) into the $\mathrm{CsPbCl}_{0.9} \mathrm{Br}_{2.1}$ perovskite to form a quasi-2D perovskite thin film, in which PEABr could efficiently passivate the traps in perovskite thin films, substantially improving their PL QYs from $0.15 \%$ to $27 \%$. Consequently, a highly efficient blue PeLED with a maximum $\eta_{\mathrm{EQE}}$ of $5.7 \%$ and a peak luminance of $3780 \mathrm{~cd} \mathrm{~m} \mathrm{~m}^{-2}$ at an EL peak of $480 \mathrm{~nm}$ was successfully demonstrated. More importantly, the operation lifetime of the 
(a)
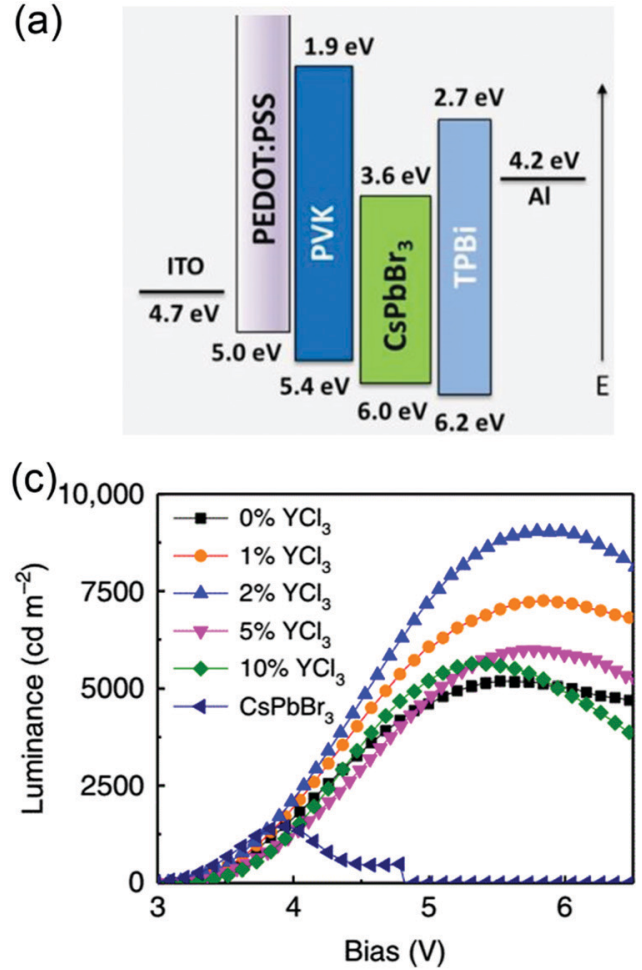
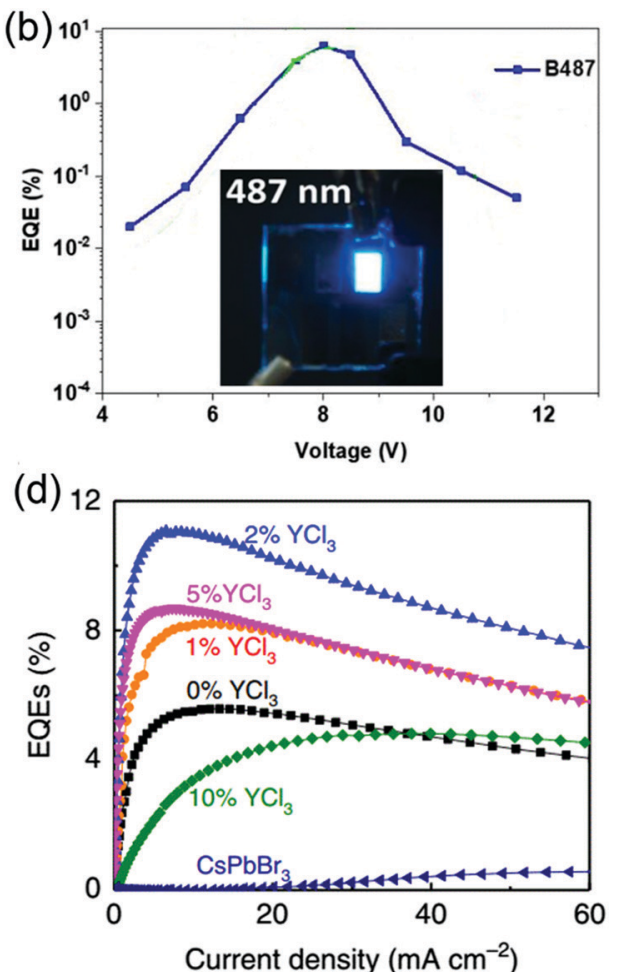

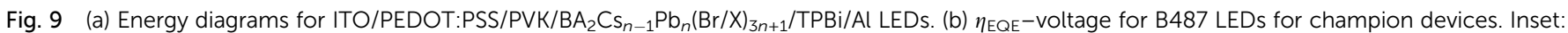

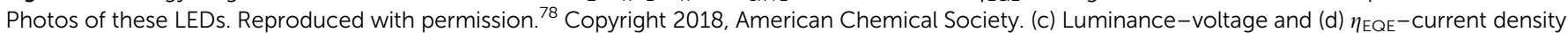
curves of $\mathrm{CsPbBr}_{3}$ : PEACl (1:1) devices with different ratios of $\mathrm{YCl}_{3}$. Reproduced with permission. ${ }^{81} \mathrm{Copyright} 2019$, Nature Publishing Group.

devices was studied under a constant applied voltage of $4.4 \mathrm{~V}$, achieving a half lifetime of around $10 \mathrm{~min}$, which is one of the best results among the reported lifetimes of blue-emitting perovskite LEDs. However, the EL spectrum of the device continuously shifted from $480 \mathrm{~nm}$ to $488 \mathrm{~nm}$ during the operating time of $9 \mathrm{~min}$, which can be attributed to the possible phase separation and other degradation processes that require further investigation. ${ }^{79}$ In addition, a considerable amount of excess cations of $\mathrm{PBA}^{+}$is usually used to avoid over-growth of the all-bromide perovskite crystals; however, the insulating of $\mathrm{PBABr}$ can impede charge injection into the perovskite films and influence the energetic coupling between the quasi-2D phases and the quantum-confined nanoparticles. Hence, Liu et al. used ethyl acetate as an antisolvent, which dissolved PBABr and did not damage the perovskite layer or the polymer layers underneath, to deposit the perovskite layer from a precursor solution of $\mathrm{PBABr}$, formamidinium bromide ( $\mathrm{FABr}$ ), $\mathrm{CsBr}$, and $\mathrm{PbBr}_{2}$ (1.1: $0.3: 0.7: 1$ molar ratio) dissolved in dimethyl sulfoxide. A symmetric peak centered at $483 \mathrm{~nm}$ with a narrow FWHM of $26 \mathrm{~nm}$ and a high PL QY of $60 \%$ was obtained, and highperformance PeLEDs with a record $\eta_{\mathrm{EQE}}$ of $9.5 \%$ and a half lifetime of $250 \mathrm{~s}$ at an initial brightness of $100 \mathrm{~cd} \mathrm{~m}^{-2}$ were successfully demonstrated using these perovskites as emitting materials. This result showed the bright outlook of blue PeLEDs for lighting and display applications. However, the maximum luminance was only $700 \mathrm{~cd} \mathrm{~m}^{-2} \cdot{ }^{80}$ To date, a record efficiency of $11.0 \%$ and a maximum luminance of $9040 \mathrm{~cd} \mathrm{~m}^{-2}$ were obtained in sky-blue PeLEDs by blending phenylethylammonium chloride
(PEACl) into $\mathrm{CsPbBr}_{3}$ to form a mixture of $2 \mathrm{D}$ and 3D perovskites, followed by the introduction of yttrium(III) chloride $\left(\mathrm{YCl}_{3}\right)$ into the mixture to further enhance the PL QY from 19.8\% to $49.7 \%$ (Fig. 9c and d). The incorporation of $\mathrm{YCl}_{3}$ served the following three functions: (1) reducing the nonradiative recombination centers in $\mathrm{PEACl}: \mathrm{CsPbBr}_{3}(1: 1)$ films, induced by accumulating $\mathrm{Y}$ on the film surfaces or grain boundaries; (2) improving spectrum stability by drastically suppressing ion migration in these films; (3) increasing the bandgap of the grain shell, which confined the charge carriers inside grains for efficient radiative recombination. Furthermore, a blue PeLED with an $\eta_{\mathrm{EQE}}$ of $4.8 \%$ was also obtained by adding $10 \% \mathrm{YCl}_{3}$ in the perovskite film. This work provides a new method to improve PL QYs in $\mathrm{Cl}$ :Br-based perovskites and related PeLEDs. ${ }^{81}$

\subsection{All-inorganic PeLEDs}

Compared to hybrid organic-inorganic halides, all-inorganic perovskites exhibit higher stability and have huge potential in various optoelectronic applications. The first research attempt on blue all inorganic PeLEDs was made by Song et al., who adopted the hot-injection method to synthesize high-quality blue $\mathrm{CsPb}\left(\mathrm{Br}_{1-x} \mathrm{Cl}_{x}\right)_{3}$ QDs with a PL QY ranging between $60 \%$ and $90 \%$ and a narrow FWHM of $23 \mathrm{~nm}$. The related PeLEDs based on PVK as the HTL and TPBi as the ETL exhibited a turn-on voltage of $5.1 \mathrm{~V}$ (at $1 \mathrm{~cd} \mathrm{~m}^{-2}$ ), a peak $\eta_{\mathrm{EQE}}$ of $0.07 \%$, a maximum brightness of $742 \mathrm{~cd} \mathrm{~m}^{-2}$, and bright blue emission $(\lambda=455 \mathrm{~nm}){ }^{82}$ Furthermore, in 2017, Yao et al. demonstrated the high brightness of blue all-inorganic PeLEDs with an emission peak of $470 \mathrm{~nm}$ and a 
narrow FWHM by directly mixing $\mathrm{CsPbBr}_{3}$ and $\mathrm{CsPbCl}_{3} \mathrm{NCs}$ dispersed in appropriate solvents. XRD, XPS, and UPS tests confirmed the production of a new-phase $\mathrm{CsPbBr}_{x} \mathrm{Cl}_{3-x}$, where $\mathrm{Br}^{-}$was partially replaced by $\mathrm{Cl}^{-}$. The resulting PeLEDs that utilized $\mathrm{NiO}_{x}$ as the HTL exhibited an enhanced current efficiency of $0.18 \mathrm{~cd} \mathrm{~A}^{-1}$ and a maximum luminance of $350 \mathrm{~cd} \mathrm{~m} \mathrm{~m}^{-2}$. $^{3}$ However, severe emission quenching was observed at the $\mathrm{NiO}_{x} /$ $\operatorname{CsPb}\left(\mathrm{Br}_{x} \mathrm{Cl}_{1-x}\right)_{3}$ interface, which could be suppressed using TFB/ PFI (perfluorinated ionomer) instead of $\mathrm{NiO}_{x}$. Such a modification can further improve the $\eta_{\mathrm{EQE}}$ to $0.5 \%$ at an emission wavelength of $469 \mathrm{~nm} .{ }^{84}$ However, the highest $\eta_{\mathrm{EQE}}$ reported is still less than $1 \%$, which is partly due to the QDs capped with relatively insulating long ligands that are required for the processing and stability of the QDs. Maybe replacing these long ligands (usually oleylamine (OAm) and oleic acid (OA)) with shorter ligands can be an effective method to realize efficient blue PeLEDs. Pan et al. capped the synthesized $\mathrm{CsPbBr}_{x} \mathrm{Cl}_{3-x} \mathrm{NCs}$ with didodecyl dimethyl ammonium bromide chloride (DDABC) via a ligand-exchange strategy that included an intermediate step to desorb protonated OAm and achieved a sky-blue PeLED with a turn-on voltage of $3.0 \mathrm{~V}$ and a maximum $\eta_{\mathrm{EQE}}$ of $1.9 \%$. This work represented a major leap for perovskites and paved the way to further exploit PeLEDs in optoelectronics through judicious surface engineering. ${ }^{85}$

The above reports on blue $\mathrm{CsPbX}_{3}$ perovskite NCs were all realized by incorporating chlorine into bromine-based perovskites to tune the bandgap. Nevertheless, these mixed-halide perovskites exhibit intrinsic phase instability, particularly under light and electrical potential, which easily produces halide migration. ${ }^{86}$ Consequently, the EL spectra of the corresponding PeLEDs spontaneously transform from blue to green within minutes under device operation. ${ }^{87-89}$ Thus, to prevent EL transformation caused by halide migration, the shape control of colloidal NCs and doping of NCs are used to adjust their optical and electronic properties. For example, $\mathrm{Xu}$ et al. developed a shape-controlled synthesis of blue-emission $\mathrm{CsPbBr}_{3}$ perovskite NCs at a relatively low temperature $\left(90^{\circ} \mathrm{C}\right)$ by tuning the amounts of oleic acid (OA) and oleylamine (OM). When they directly added $0.6 \mathrm{~mL}$ of $\mathrm{OA}$ and $0.3 \mathrm{~mL}$ of OM into the reaction system, monodispersed, spherical QDs with a narrow PL emission peak at $453 \mathrm{~nm}$ were obtained. By adjusting the amounts of $\mathrm{OA}$ and $\mathrm{OM}$ from $0.3 \mathrm{~mL}$ of $\mathrm{OA}$ and $0.7 \mathrm{~mL}$ of $\mathrm{OM}$ and $0.5 \mathrm{~mL}$ of $\mathrm{OA}$ and $0.5 \mathrm{~mL}$ of $\mathrm{OM}$ to $0.8 \mathrm{~mL}$ of $\mathrm{OA}$ and $0.2 \mathrm{~mL}$ of OM, they acquired lamellar-structured 0D QDS and face-to-face stacking $2 \mathrm{D}$ nanoplatelets in addition to flatlying 2D nanosheets with PL emissions at 472, 449, and $452 \mathrm{~nm}$, respectively. This is different from the original green emission ( $\sim 510 \mathrm{~nm}$ ) of $\mathrm{CsPbBr}_{3}$ nanocubes, which may be ascribed to the strong quantum confinement effects of these various nanoshapes. Such superior shape-controlled and PL-tunable merits made them favorable for optoelectronic devices, particularly for blue PeLEDs. ${ }^{90}$ Furthermore, the doping of $\mathrm{Al}^{3+}, \mathrm{Cu}^{2+}, \mathrm{La}^{3+}$, and $\mathrm{F}^{-}$ions as co-dopant can provide $\mathrm{CsPbBr}_{3}$ NCs with a strong blue emission. ${ }^{91-93}$ For instance, the doping of $\mathrm{Cu}^{2+}$ ions into the perovskite lattice to form $\mathrm{CsPb}_{1-x} \mathrm{Cu}_{x} \mathrm{X}_{3}$ QDs could not only improve their thermal stability but also enhance the PL QY from $23 \%$ to more than $80 \% .{ }^{92}$ However, the doped blue NCs have not been applied to PeLEDs. Previous reports confirmed that Mn-doping could significantly increase the PL QY of the perovskite film and enhance the stability of $\mathrm{CsPbBr}_{3} \mathrm{NCs}$ in green PeLEDs. ${ }^{94}$ However, these Mn-doping sites could be efficient emitters in their own right. Hence, Congreve et al. adopted a two-step sequential synthesis approach to carefully tune the Mn-doping to obtain an emission peak of $\sim 468 \mathrm{~nm}$ while preventing significant Mn emission. Fig. 10a and b show that Mn-doping could increase PL QYs from 9\% for undoped films to $28 \%$ for the $0.19 \%$ doped sample. In addition, a significant increase in emissive state lifetime was observed after achieving $0.19 \%$ PL QY using the Mn dopant, indicating a reduction in trap-state densities. To identify the engineering benefits of this material improvement, Congreve et al. fabricated PeLEDs with PEDOT:PSS and TFB/PFI as the HIL/HTL together with TPBi as the ETL, and all samples exhibited pure blue emission only from the perovskite emitter layer (see Fig. 10c). In particular, up to four-fold improvement in $\eta_{\mathrm{EQE}}$ (reaching a maximum of $2.12 \%$ ) was obtained with $0.19 \%$ Mn-doping, which, to the best of our knowledge, is the highest value at this wavelength range for all-inorganic PeLEDs (Fig. 10d). Mn-Doping provides a straightforward method to increase the performance of blue-emitting PeLEDs. ${ }^{95}$

\section{Heavy-metal-free QD-LEDs}

The above described QD-LEDs mostly depend on Cd-based or Pb-based QDs, and their intrinsic toxicity limits their further commercialization because of strict environmental regulations such as the Restriction of Hazardous Substances (RoHS) directive in Europe. Hence, heavy-metal-free QD-LEDs are desirable in future displays and lighting. Thus far, several heavy-metal-free material systems have been developed, including ZnSe-based, InP-based, Cu-based and carbon dot (CD)-based QD-LEDs. The recent advances in heavy-metal-free blue QD-LEDs are summarized in Table 3.

\subsection{ZnSe-based QD-LEDs}

Owing to the large bandgap of $2.7 \mathrm{eV}$, ZnSe can be large sized to obtain relevant blue color wavelength. The first blue/violet QD-LED based on Cd-free ZnSe/ZnS QDs with the PL peak located at $420 \mathrm{~nm}$ was reported by Xiang et al., delivering a reduced turn-on voltage of $3.5 \mathrm{~V}$ and a maximum $\eta_{\mathrm{EQE}}$ of $0.65 \%$ with PVK as the HTL. The achieved $\eta_{\mathrm{EQE}}$ of $0.65 \%$ suggested that Cd-free ZnSe/ZnS QD-LEDs would be a promising candidate for short-wavelength LEDs. $^{96}$ In the following year, Ji et al. demonstrated color-saturated deep-blue emission with a narrow FWHM of $15 \mathrm{~nm}$ at $\lambda=441 \mathrm{~nm}$ in an inverted structure, the maximum brightness and current efficiency of which could

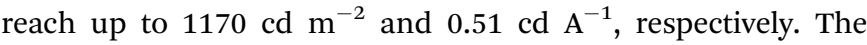
poor device performance may be attributed to the low PL QYS and stability of QDs in the violet-blue area. ${ }^{97}$ For improving the PL QY of ZnSe/ZnS core/shell QDs and the performance of the related QD-LEDs, Shen et al. adopted "a low temperature injection and high temperature growth" method together with the octanethiol ligand to synthesize nearly monodispersed 

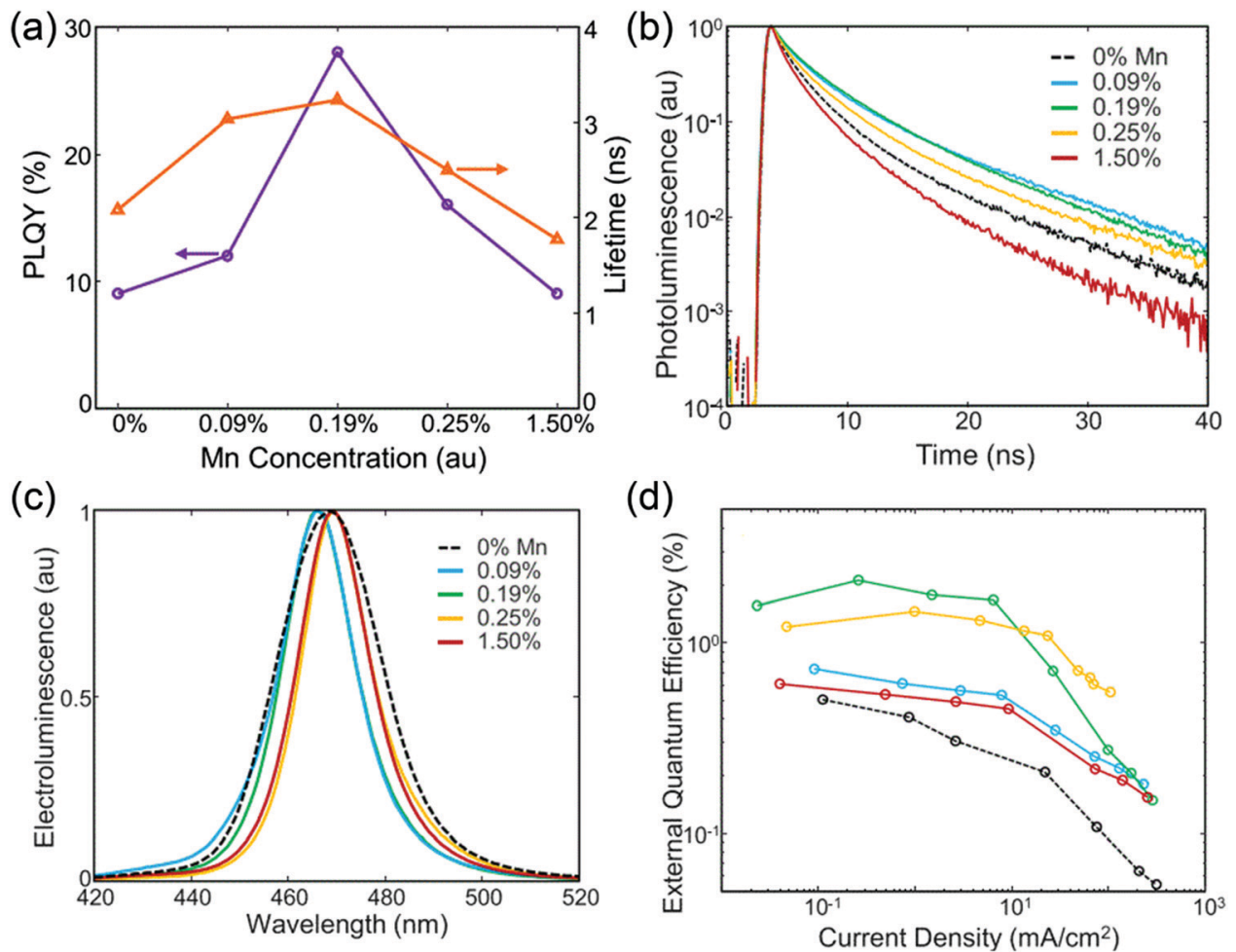

Fig. 10 (a) PL QY and 1/e lifetime of the perovskite emission of thin films of NCs. (b) Time-resolved perovskite luminescence of the different NC species. (c) EL spectra of the fabricated PeLEDs with NCs of varying Mn content. (d) $\eta_{\text {EQE }}$ as a function of current density for the fabricated PeLEDs. Reproduced with permission. ${ }^{95}$ Copyright 2018, Elsevier Ltd.

Table 3 Recent advances in heavy-metal-free blue QD-LEDs

\begin{tabular}{|c|c|c|c|c|c|c|c|c|}
\hline QDs & Year & Device structure & PL peak $(\mathrm{nm})$ & $\eta_{\mathrm{EQE}}(\%)$ & $\eta_{\mathrm{A}}\left(\mathrm{cd} \mathrm{A}^{-1}\right)$ & $V_{\text {on }}(\mathrm{V})$ & $L_{\max }\left(\mathrm{cd} \mathrm{m}^{-2}\right)$ & Ref. \\
\hline \multirow[t]{4}{*}{ ZnSe-based QDs } & 2012 & ITO/PEDOT:PSS/PVK/ZnSe/ZnS/ZnO/Al & 420 & 0.65 & - & - & - & 96 \\
\hline & 2013 & ITO/ZnO/ZnSe/ZnS/TCTA/ $\mathrm{MoO}_{3} / \mathrm{Al}$ & 441 & - & 0.51 & 4.0 & 1170 & 97 \\
\hline & 2015 & ITO/PEDOT:PSS/PVK/ZnSe/ZnS/ZnO/Al & 425 & 7.83 & 1.38 & 7.0 & 2623 & 98 \\
\hline & 2019 & ITO/PEDOT:PSS/PVK/ZnSeTe/ZnSe/ZnS/ZnMgO/Al & 441 & 4.2 & 2.4 & 4 & 1195 & 102 \\
\hline \multirow[t]{2}{*}{ InP-based QDs } & 2017 & ITO/ZMO/InP/ZnS//ZnS/CBP/ $\mathrm{MoO}_{3} / \mathrm{Al}$ & 477 & - & - & - & 90 & 107 \\
\hline & 2020 & ITO/PEDOT:PSS/TFB/InP/GaP/ZnS//ZnS/ZnO/Al & 488 & 1.01 & - & 2 & 3120 & 108 \\
\hline \multirow[t]{3}{*}{ Cu-based QDs } & 2014 & ITO/PEDOT:PSS/PVK/CGS/ZnO/Al & 479 & 0.007 & 0.01 & - & 39 & 115 \\
\hline & 2019 & ITO/PEDOT:PSS/PVK/CZGS:Mn/ZnS/ZMO/Al & 470 & 4.2 & 2.3 & 4.9 & 1352 & 117 \\
\hline & 2019 & ITO/PEDOT:PSS/PVK/ZCGS/ZnS/ZMO/Al & 474 & 7.1 & 11.8 & 4.0 & 1404 & 118 \\
\hline \multirow[t]{5}{*}{ Carbon dots } & 2013 & ITO/PEDOT:PSS/poly-TPD/CDs/TPBi/LiF/Al & 420 & - & - & 5 & 24 & 120 \\
\hline & 2016 & ITO/PEDOT:PSS/CDs/TPBi/Ca/Al & 430 & - & 0.084 & 4.7 & 136 & 121 \\
\hline & 2018 & ITO/PEDOT:PSS/PVK:CDs/TPBi/LiF/Al & 467 & - & - & 8.5 & 569.8 & 122 \\
\hline & 2018 & ITO/PEDOT:PSS/PVK:CDs/TPBi/Ca/Al & 472 & - & 1.22 & 4.3 & 1882 & 123 \\
\hline & 2019 & ITO/PEDOT:PSS/TFB/PVK:CDs/TPBi/LiF/Al & 433 & 4.0 & 2.6 & - & 5240 & 124 \\
\hline
\end{tabular}

ZnSe/ZnS core/shell QDs with high PL QYs of more than $80 \%$, high color purity with a FWHM of about $12-20 \mathrm{~nm}$, and good color tunability in the violet-blue range from 400 to $455 \mathrm{~nm}$ achieved by adjusting the reaction time and temperature of the shell (Fig. 11a). Significantly, such ZnSe/ZnS core/shell QDs exhibited very good chemical/photochemical stability under ultraviolet (UV)-irradiation and repeated purification processes, which was essential for the high performance of QD-LEDs; thus, the highly bright violet QD-LEDs were successfully demonstrated with a fully solution-processed method, exhibiting a maximum luminance of $2632 \mathrm{~cd} \mathrm{~m}^{-2}$ and a peak $\eta_{\mathrm{EQE}}$ of $7.83 \%$ with the EL peak at $437 \mathrm{~nm}$ (Fig. 11b). ${ }^{98}$ However, their EL wavelength (437 nm) was quite beyond the scope of the desired blue color (440-460 nm). To obtain ZnSe/ZnS QDs with the PL peak reaching $460 \mathrm{~nm}$, the size of ZnSe/ZnS QDs should be sufficiently large to render the quantum confinement effect negligible. To date, the size control of ZnSe QDs has been implemented simply by varying core reaction conditions (e.g., time and temperature). ${ }^{99,100}$ Analogous to InGaP QDs, ${ }^{101}$ an alternative strategy toward blue emission from ZnSe QDs is compositional alloying of 
(a)
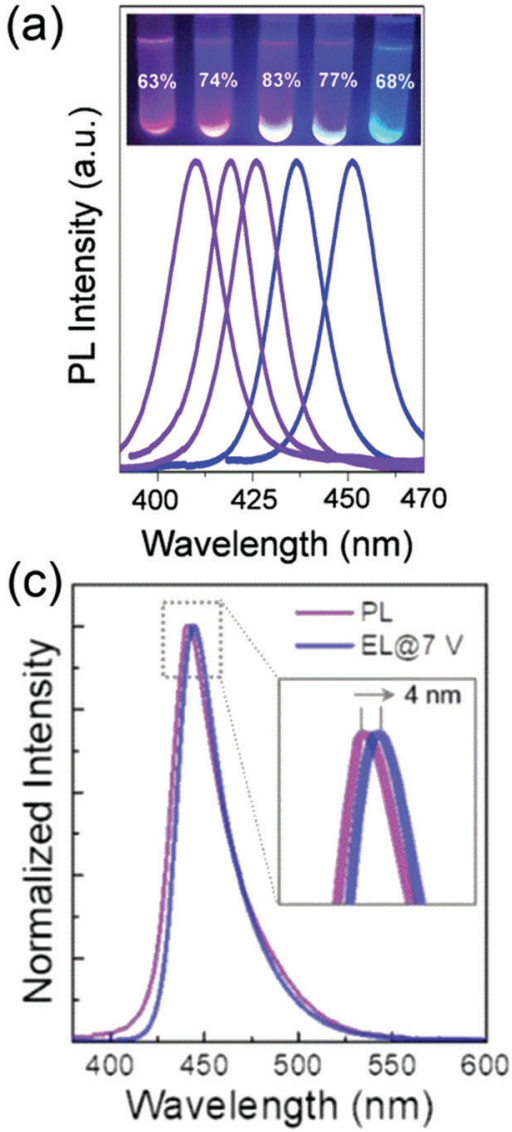

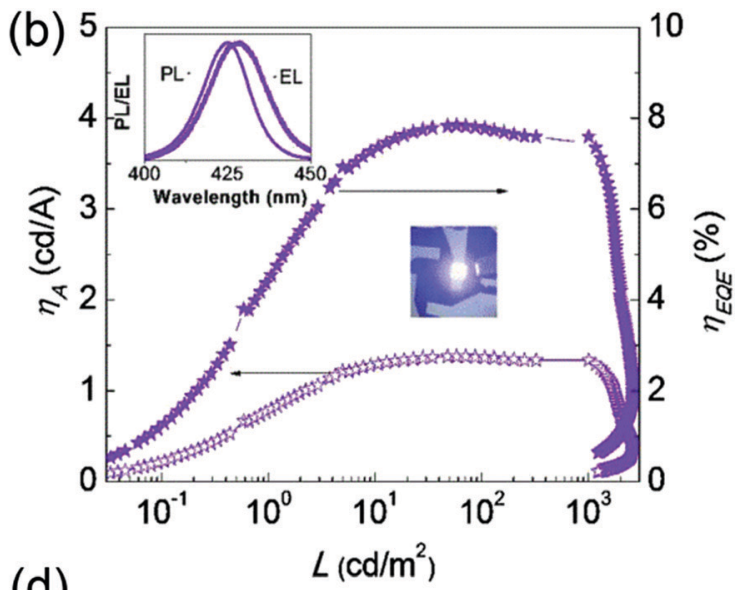

(d)

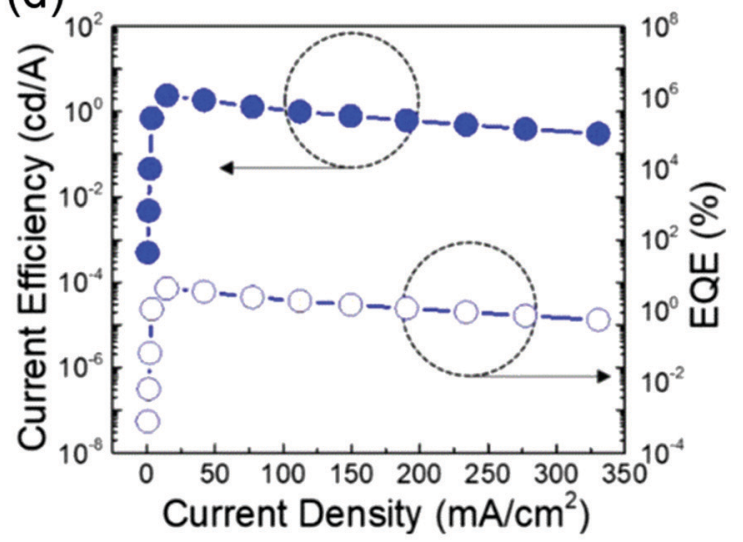

Fig. 11 (a) Normalized PL spectra of ZnSe/ZnS core/shell QDs (dispersed in hexane) and representative emission colors of ZnSe/ZnS core/shell QDs (in hexane) under the radiation of an ultraviolet lamp. (b) Current efficiency- $\eta_{E Q E}-L$ characteristics for the ZnSe/ZnS-based QD-LEDs. Reproduced with permission. ${ }^{98}$ Copyright 2015, Royal Society of Chemistry. (c) Spectral comparison of the PL of a dilute ZnSeTe/ZnSe/ZnS QD dispersion with the EL collected at 7 V. (d) Current efficiency and $\eta_{\text {EQE }}$ as a function of current density for QD-LEDs with an ITO/PEDOT:PSS/PVK/ZnSeTe/ZnSe/ZnS QDs/ ZnMgO/Al structure. Reproduced with permission. ${ }^{102}$ Copyright 2019, American Chemical Society.

high-bandgap ZnSe with low-bandgap ZnTe in QD synthesis. Yang et al. developed a heat-up synthesis of alloyed ZnSeTe QDs, and further introduced inner and outer shells of ZnSe and $\mathrm{ZnS}$, respectively, to form double-shelled ZnSeTe/ZnSe/ZnS QDs. As shown in Fig. 11c, the resulting blue (441 nm)emission QDs with an optimal Te/Se ratio in the ZnSeTe core and ZnSe inner shell thickness possessed a high PL QY of 70\% and a narrow FWHM of $32 \mathrm{~nm}$. Finally, owing to the suppressed nonradiative processes of Förster energy transfer and Auger recombination with the presence of relatively thick double shells and ZnSe inner shell, the blue ZnSeTe QD-LEDs that utilized such QDs exhibited a peak luminance of $1195 \mathrm{~cd} \mathrm{~m}^{-2}$ and an $\eta_{\mathrm{EQE}}$ of $4.2 \%$ (Fig. 11d), which is the highest device performance to date among the non-Cd blue QD-LEDs. This work will pave the way for the realization of next-generation non-Cd QD-LED displays in the future. ${ }^{102}$

\subsection{InP-based QD-LEDs}

Among the non-Cd QDs, InP QDs have been highlighted as the most promising alternatives to CdSe QDs because of their increasingly enhanced PL characteristics in terms of PL line width and PL QY, mainly enabled by an elaborate control of InP core size distribution and rational multi-shell-based heterostructural engineering. ${ }^{103-105}$ Following years of hard work on the optimization of emitting materials, device architecture, and deep understanding of the operational mechanism, the $\eta_{\mathrm{EQE}}$ of red and green InP-based QD-LEDs has exceeded 12\% and 13\%, respectively; ${ }^{25,26}$ however, their blue-emitting counterpart has been challenging because of a relatively small bulk bandgap (1.35 eV at room temperature) of InP QDs. ${ }^{106}$ In 2017, Shen et al. synthesized high PL QY (up to 76\%) InP/ZnS small-core/ thick-shell tetrahedral-shaped QDs for blue QD-LEDs for the first time. However, the maximum luminance was only $90 \mathrm{~cd} \mathrm{~m}^{-2}$, and no other EL characteristics were reported. ${ }^{107}$ The poor device performance may be attributed to the low PL QY of the QD assembled emitting thin film as well as the low carrier injection and transport process. Although an extra thick ZnS shell on the surface of an InP core can enhance the PL QY, ultimately suppressing the nonradiative Förster resonance energy transfer (FRET) processes between small sized QDs, the large lattice mismatch between InP and $\mathrm{ZnS}(\sim 7.85 \%)$ will lead to the lattice collapse of InP QDs. So, in 2020, Shen et al. introduced a thin GaP bridging layer between the inner InP core and the outer ZnS shell through the "low temperature nucleation and high 
temperature growth" method, which significantly reduced the impact of the large lattice mismatch. By further coating of the ZnS thick shell, the resultant InP/GaP/ZnS//ZnS QDs showed a large particle size of $7.0 \pm 0.9 \mathrm{~nm}$ and a high PL QY of $81 \%$. The corresponding QD-LEDs exhibited a record brightness and $\eta_{\mathrm{EQE}}$ of $3120 \mathrm{~cd} \mathrm{~m}^{-2}$ and $1.01 \%$, respectively. Using large-scale density functional theory (DFT) calculations on thousand-atom QDs, Shen et al. found that thicker-shell ones favor a more balanced carrier injection and simultaneously suppress the FRET between closely packed QDs, which collectively improve the performances of blue InP-based QD-LEDs. ${ }^{108}$ In addition, reports are available on blue InP-based QDs based on changing the ratio of InP:ZnS, reaction conditions, types of precursors and surface ligands, or even doping Ga ions. ${ }^{109-112}$ For example, Ramasamy et al. synthesized highly luminescent $\operatorname{In}(\mathrm{Zn}) \mathrm{P} / \mathrm{ZnSe} /$ ZnS QDs with tunable emission from 488 to $641 \mathrm{~nm}$ and high color purity by synthesizing Zn carboxylate covered In(Zn)P QDS in a single step, and the FWHM and PL QY were $35 \mathrm{~nm}$ and $44 \%$, respectively, for blue-sky emission $(488 \mathrm{~nm}) .{ }^{113}$ In addition, blue-emitting InP/ZnS core/shell QDs with a PL peak of $475 \mathrm{~nm}$ and a FWHM of $39 \mathrm{~nm}$ were demonstrated by decreasing the reaction temperature after mixing the precursors. ${ }^{114}$ However, the PL peak of these QDs is mostly higher than $470 \mathrm{~nm}$, and they have no application in QD-LEDs. Hence, the performance of pure blue InP-based QD-LEDs needs to be further improved.

\subsection{Cu-based QD-LEDs}

Cu-based QDs are another promising class of non-Cd emitting materials. By controlling composition, size, and cationic offstoichiometry, the PL emission of these QDs can be tuned from green to deep red. However, the emitting wavelengths of I-III-VI QDs shorter than $500 \mathrm{~nm}$ are rarely reported. Recently, a rather wider tunability has been accomplished primarily through alloying $\mathrm{Cu}-\mathrm{In}-\mathrm{S}$ QDs with a higher bandgap material of $\mathrm{ZnS}$ or $\mathrm{Cu}-\mathrm{Ga}-\mathrm{S}$ to produce quaternary $\mathrm{Zn}-\mathrm{Cu}-\mathrm{In}-\mathrm{S}$ or $\mathrm{Cu}-\mathrm{In}-\mathrm{Ga}-\mathrm{S}$ ones. For the first time, Yang et al. demonstrated a sky-blue $\mathrm{Cu}-\mathrm{Ga}-\mathrm{S} / \mathrm{ZnS}$ QD-based QD-LED having a luminance of $39 \mathrm{~cd} \mathrm{~m}^{-2}$ and an $\eta_{\mathrm{EQE}}$ of $0.007 \%$. The poor performance may be due to the low PL QY $(\sim 20 \%)$ of $\mathrm{Cu}-\mathrm{Ga}-\mathrm{S} / \mathrm{ZnS}$ QDs. ${ }^{115}$ The same group also optimized $\mathrm{Cu}-\mathrm{Ga}-\mathrm{S} / \mathrm{ZnS}$ QDs via $\mathrm{Zn}$ alloying, and the resulting $\mathrm{Zn}-\mathrm{Cu}-\mathrm{Ga}-\mathrm{S} / \mathrm{ZnS}$ core/shell QDs possessed tunable emissions in the azure-to-blue range and a high PL QY of 78-83\% with different amounts of the Zn precursor (Fig. 12a and b). Nevertheless, no report is available on the related QD-LEDs. ${ }^{116}$ Based on bluish-emitting double-shelled QDs of $\mathrm{Zn}-\mathrm{Cu}-\mathrm{Ga}-\mathrm{S} / \mathrm{ZnS} / \mathrm{ZnS}$, a series of Mn doped $\mathrm{Zn}-\mathrm{Cu}-\mathrm{Ga}-\mathrm{S}$ QDs with various $\mathrm{Mn} / \mathrm{Cu}$ molar ratios were synthesized via surface adsorption and lattice diffusion. Regardless of the concentration of Mn-doping, all QDs possessed high PL QYs ranging from $74 \%$ to $79 \%$. Similar to the Mn-doped Cd-based QDs, the resulting Mn-doped $\mathrm{Zn}-\mathrm{Cu}-\mathrm{Ga}-\mathrm{S}$ QDs exhibited a distinct $\mathrm{Mn}^{2+}{ }^{4} \mathrm{~T}_{1}-{ }^{6} \mathrm{~A}_{1}$ emission simultaneously with host intragap states involving emissions of free-to-bound and donoracceptor pair (DAP) recombinations. However, different from Mn-doped Cd-based QD-LEDs, Mn emission was nearly absent in the EL of the Zn-Cu-Ga-S:Mn/ZnS/ZnS based QD-LED (Fig. 12d), which may be attributed to a preferential hole injection from the HTL to the QD emitting layer and subsequent rapid trapping of holes at acceptor states. When these QDs were incorporated into QD-LEDs, the related device with $\mathrm{Mn} / \mathrm{Cu}=16$ exhibited a high luminance of $1352 \mathrm{~cd} \mathrm{~m}^{-2}$, a peak current efficiency of $2.3 \mathrm{~cd} \mathrm{~A}^{-1}$, and a maximum $\eta_{\mathrm{EQE}}$ of $4.2 \%$ (Fig. 12c). ${ }^{117}$ Despite these parameters, $\mathrm{Cu}$-In-S-based QDs with Mn-doping require detailed adjustment of the $\mathrm{Mn}$ concentration, increasing the complexity of the synthesis. Therefore, after two years of hard work, Yang et al. reported non-Cd $\mathrm{Zn}-\mathrm{Cu}-\mathrm{Ga}-\mathrm{S} / \mathrm{ZnS}$ QDs with a high PL QY of $80 \%$ and a PL peak at $474 \mathrm{~nm}$. The related QD-LEDs demonstrated a record $\eta_{\mathrm{EQE}}$ of $7.1 \%$ and a peak current efficiency of $11.8 \mathrm{~cd} \mathrm{~A}^{-1}$ by all-solution-processed fabrication, corresponding to the record quantities among non-Cd QD-based blue EL devices reported to date. This work accelerates the commercialization of non-Cd QD LEDs. ${ }^{118}$

\subsection{Carbon dot-based QD-LEDs}

CDs generally comprise abundant elements and are free of heavy metals. Since the first synthesis of CDs reported by Sun et al. in $2006,{ }^{119}$ these materials have attracted widespread research attention owing to their tunable luminescence emission, high resistance to photobleaching, and intrinsic nontoxicity. In recent years, CDs have been demonstrated to possess high PL QYs (up to 60-80\%), rendering them competitors in lightemitting performance. The first blue carbon-dot based LED (CD-LED) was reported by Zhang et al., who fabricated CD-LEDs by sandwiching the CD emitting layer between the organic HTL (PEDOT:PSS/poly-TPD) and the organic ETL (TPBi); this device exhibited a turn-on voltage of $5 \mathrm{~V}$ and a maximum brightness of $24 \mathrm{~cd} \mathrm{~m}^{-2}$. However, therein, the emission would transfer from blue to white light with increasing voltage up to $9 \mathrm{~V} .{ }^{120}$ Hence, to obtain pure blue emission at any voltage, Yuan et al. synthesized multicolor bandgap fluorescent CDs by simply controlling the fusion and carbonization of citric acid (CA) and diaminonaphthalene (DAN) with a unique amino-substituted rigid carbon skeleton structure. The as-prepared CDs were nitrogen-doped and had a high PL QY of up to 75\% for blue emission, a high degree of crystallinity, and high surface passivation. Consequently, the pure blue LEDs were realized by directly using these CDs as the emitting layer without the HTL, and these blue LEDs exhibited voltageindependent emission with an increased luminance of $136 \mathrm{~cd} \mathrm{~m}^{-2}$. This work served the purpose of understanding and providing significant opportunities for further improvement in CD-LEDs. ${ }^{121}$ In 2018, Xu et al. adopted a strategy of host-guest doping to fabricate blue CD-LEDs using the oleophilic CDs (guest) doped in PVK (host) as the emitting layer because of the good co-solubility of oleophilic CDs and PVK in chlorobenzene at any doping concentration. Thus, the fabricated CD-LEDs obtained an ultrahigh brightness of $569.8 \mathrm{~cd} \mathrm{~m}^{-2}$ with pure blue emission that predominantly originated from the emission of CDs by the hostguest energy transfer mechanism. This result demonstrated that oleophilic CDs were conducive to fabricating CD-LEDs with both high brightness and pure emission. However, the turn-on voltage of CD-LEDs was $8.5 \mathrm{~V}$, which might be due to the deep HOMO of these CDs, reaching up to $-6.43 \mathrm{eV} \cdot{ }^{122}$ Moreover, the FWHM of 
(a)
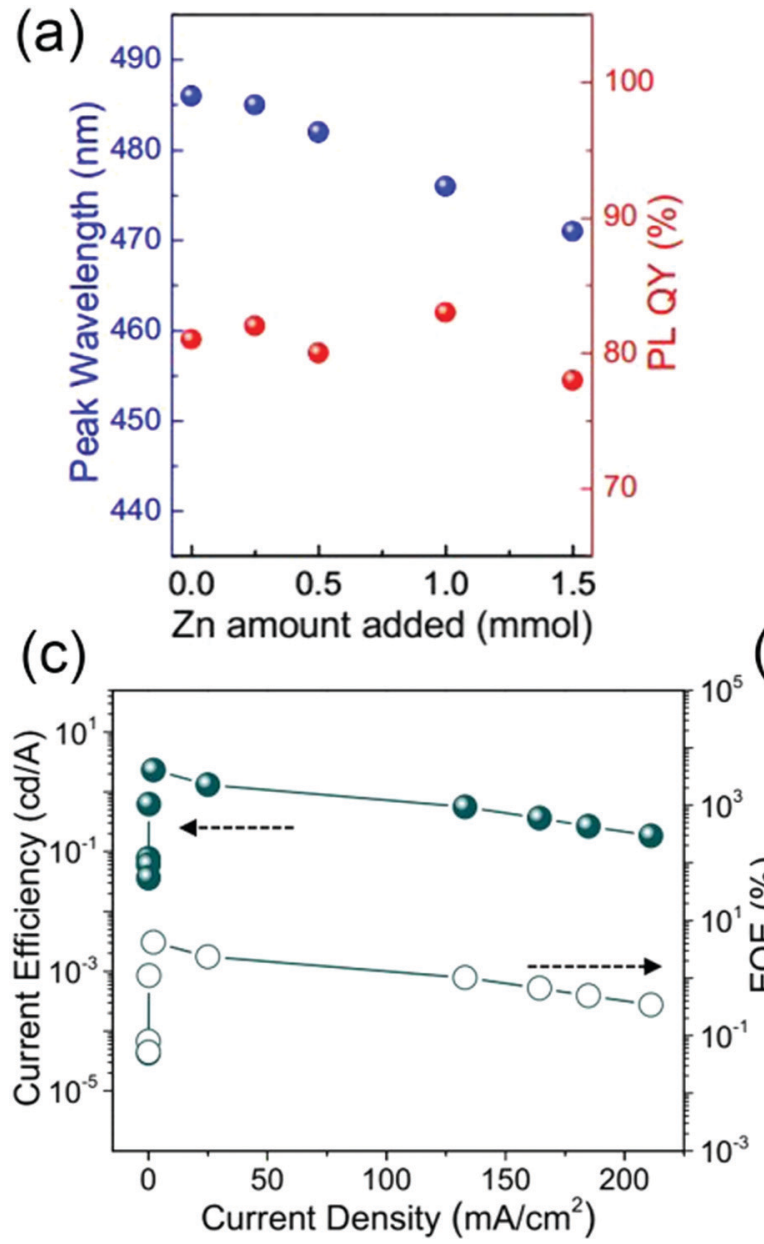

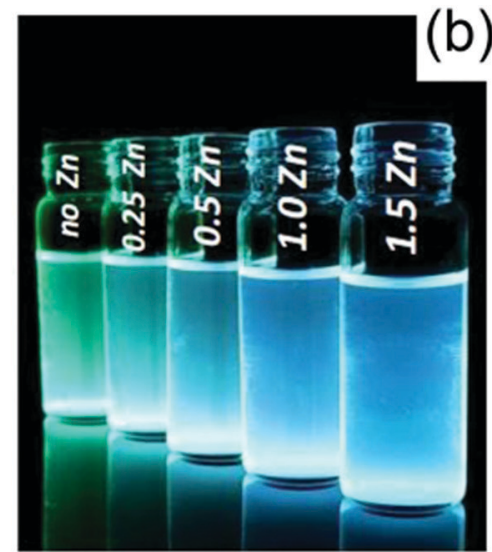

(b)

$(\mathrm{d})$

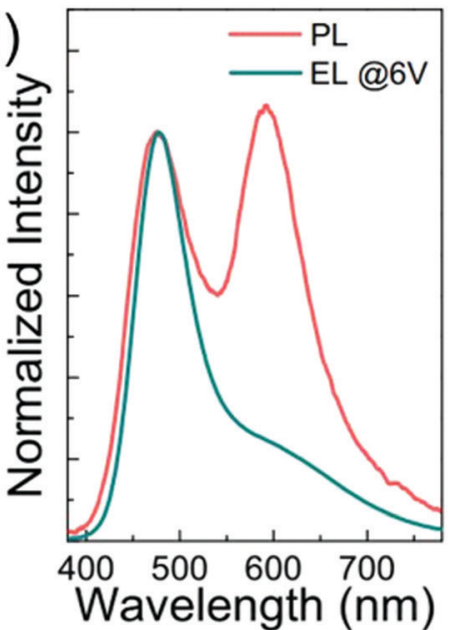

Fig. 12 (a) PL peak-PL QY of azure-to-blue-emitting Zn-Cu-Ga-S/ZnS QDs synthesized with different amounts of the Zn precursor. (b) Photograph of highly fluorescent QD dispersions under UV lamp excitation. Reproduced with permission. ${ }^{116}$ Copyright 2017, Royal Society of Chemistry. (c) Current efficiency- $\eta_{\mathrm{EQE}}$-current density characteristics of the $\mathrm{Mn} / \mathrm{Cu}=16$-doped $\mathrm{QD}$-LED. (d) PL and EL (at $6 \mathrm{~V}$ ) spectra of $\mathrm{Mn} / \mathrm{Cu}=16$-doped $\mathrm{QDs}$. Reproduced with permission. ${ }^{117}$ Copyright 2019, American Chemical Society.

these CDs typically exceeds $80 \mathrm{~nm}$, which may originate from the electronic coupling to molecular vibrations and distortions, limiting their display applications. Therefore, Yuan et al. adopted the tri-molecular reaction route to design the neighboring active $-\mathrm{OH}$ and $-\mathrm{H}$ groups for six-membered ring cyclization with three-fold symmetric phloroglucinol (PG) as the reagent. Owing to the molecular purity and crystalline perfection, the as-synthesized triangular CDs exhibited high color purity with a narrow FWHM of 29-30 nm and high PL QYs of up to 54-72\% (Fig. 13a). As shown in Fig. 13b and c, when incorporating these CDs into LEDs, a high-performance device having a maximum luminance of $1882 \mathrm{~cd} \mathrm{~m}^{-2}$, a current efficiency of $1.22 \mathrm{~cd} \mathrm{~A}^{-1}$, and a low turn-on voltage of $4.3 \mathrm{~V}$ was obtained at an EL emission peak of $476 \mathrm{~nm}$. More importantly, the CD-LEDs exhibited an apparently voltage-independent emission color, indicating the high color stability of the device (Fig. 13d). ${ }^{123}$ Moreover, different oxygen-containing functional groups such as carboxyl, carbonyl, and epoxy groups at the edge or basal plane sites in the CDs, functioning as transient trap states, may also contribute to spectral broadening. To eliminate the oxygen-containing functional groups while retaining solution processability, Sargent et al. ${ }^{124}$ developed a two-step method for the synthesis of high-color-purity blue CDs, in which the CDs functionalized with $\mathrm{OH}, \mathrm{COOH}$, and $\mathrm{NH}_{2}$ were first synthesized through solvothermal treatment using citric acid and diaminonaphthalene, followed by additional surface amination using ammonia liquor and hydrazine hydrate under high temperature to further eliminate the oxygen-containing functional groups. Finally, the CDs were purified via silica column chromatography using a mixture of dichloromethane and methanol as the eluent to remove the byproducts and unreacted precursors. The as-prepared CDs exhibited a narrow FWHM of $35 \mathrm{~nm}$ and a high PL QY of $70 \pm 10 \%$, which are among the highest values for CDs reported to date. The high PL QY could be ascribed to efficient edge amination, which decreased the number of defects of CDs and thus suppressed non-radiative pathways. When incorporating these CDs blended PVK into LEDs, a maximum luminance of $5240 \mathrm{~cd} \mathrm{~m}^{-2}$, a peak $\eta_{\mathrm{EQE}}$ of $4 \%$, and a current efficiency of $2.6 \mathrm{~cd} \mathrm{~A}^{-1}$ were obtained at an EL emission peak of $440 \mathrm{~nm}$, exceeding deep-blue QD-LEDs based on $\mathrm{Cd} / \mathrm{Pb}$-comprising materials. ${ }^{50,125}$ However, the investigation of new materials as 

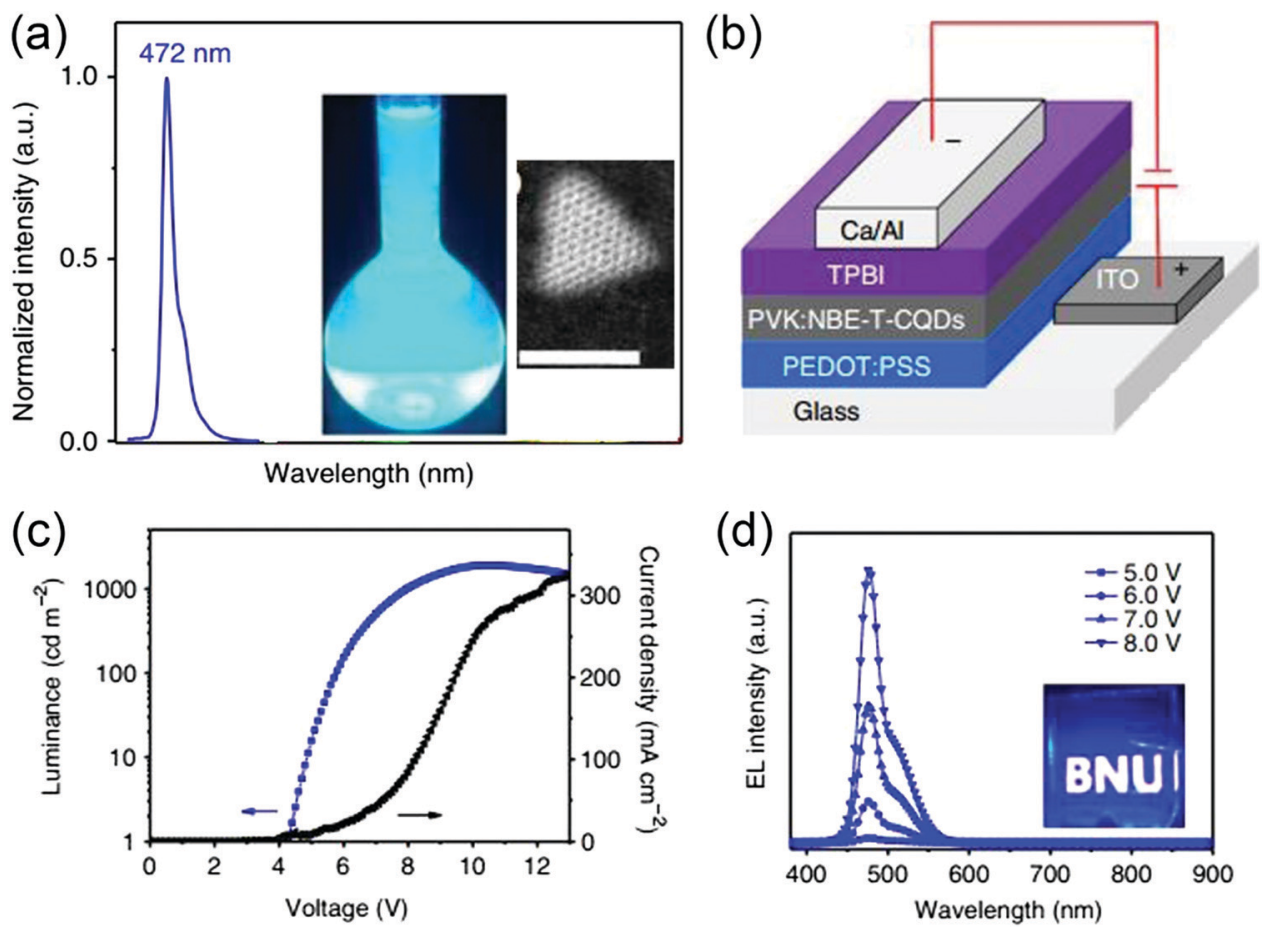

Fig. 13 (a) PL spectrum of the CDs. Insets: Photographs under UV light (left) and HAADF-STEM image of the CDs. (b) Device structure of the CD-LEDs. (c) L-J-V characteristic of the CD-LEDs. (d) EL spectra of the blue-LEDs at different bias voltages. Reproduced with permission. ${ }^{123}$ Copyright 2018, Nature Publishing Group.

the emitting layer for LEDs is at an initial stage. Hence, more optimization of charge injection (holes and electrons) and betterdesigned devices will be put forward for high-performance CD-LEDs.

\section{Conclusion and perspective}

In recent years, blue QD-LEDs have experienced major development in terms of QDs and CTL materials, device physics, and novel device structures. In this review, we studied the development of blueemitting QD-LEDs, including Cd-based QD-LEDs, PeLEDs, and heavy metal-free QD-LEDs. The Cd-based QD-LEDs could be optimized via nanostructure engineering and stable surface modification of the QD emitting layer, engineering of related device architecture, and a deep understanding of the aging mechanism of blue devices; the device's $\eta_{\mathrm{EQE}}$ and operating lifetime have increased from less than $0.1 \%$ to $19.8 \%$ and from almost negligible hours to $7000 \mathrm{~h}$, respectively. The $\eta_{\mathrm{EQE}}$ of PeLEDs has increased from less than $0.001 \%$ to $11 \%$ through structure and component optimization of perovskite QDs and by selecting the appropriate device architecture. However, compared with their red and green counterparts, the efficiency and device lifetime of blue QD-LEDs are still lagging. In addition, the development of heavy metal-free QD-LEDs is relatively slow owing to the challenge in preparing blue emitting materials. Hence, the following aspects are critical for accelerating the commercialization of blue QD-LEDs.

(1) Performance and operating lifetime of blue Cd-based QD-LEDs To date, the device efficiency of blue QD-LEDs has remained lower than that of the red and green devices (red: $\eta_{\mathrm{EQE}}=30.9 \%$, green: $\left.\eta_{\mathrm{EQE}}=25.04 \%\right) \cdot{ }^{126,127}$ Additionally, the longest operation lifetimes of Cd-based blue QD-LEDs are only on the order of $7000 \mathrm{~h}$ at an initial brightness of $100 \mathrm{~cd} \mathrm{~m}^{-2}$, which is a little inferior to the minimum requirement for display applications $(10000 \mathrm{~h})$. These poor device performances may be attributed to the relatively low PL QYs of QD films, the imbalanced charge injection/transport resulting from the large energy level offset between the QD layers and conventional organic HTLs with lower hole mobility, and the vague understanding of quenching mechanisms, such as energy transfer in blue QD films, interfacial charge transfer between blue QDs and CTLs, and electrical-fieldinduced quenching in blue QD-LEDs. ${ }^{30,31,128}$ Therefore, to develop efficient blue QDs and related QD-LEDs, intensive research is required on crystal engineering for blue QDs to have the appropriate energy-level structure, material chemistry for high-performance HTLs with simultaneous high work function and hole mobility, together with an in-depth understanding of the exciton quenching mechanism of blue QDs.

\section{(2) Stability of blue PeLEDs}

The stability and lifetime of perovskite LEDs remain a challenge, hindering their practical application. Although efforts have been directed toward improving the stability of perovskite devices by mixing inorganic Cs cations and organic cations at the A site and constructing $2 \mathrm{D}$ or quasi-2D perovskites as well as modifying the perovskite surface by appropriate ligands, exciton quenching still exists at the interface between CTLs and QDs. Currently, the best operating lifetime of blue perovskite devices is $10 \mathrm{~min}$ under a constant applied voltage of $4.4 \mathrm{~V}$, which is far from the requirement of display and lighting applications. This poor 
performance may be associated with ion migration and poor thermostability in the perovskite. Therefore, further research is required to address issues such as improving the reproducibility of perovskite devices, long-term stability, and a deep fundamental understanding of lead halide perovskite materials and their device architectures.

\section{(3) Development of heavy-metal-free blue QD-LEDs}

Despite the available high performance, Cd- and perovskitebased QDs contain toxic elements $\mathrm{Cd}$ and $\mathrm{Pb}$ whose amount should be tightly controlled in consumer electronics. Hence, synthesizing heavy-metal-free QDs is highly desirable. In this case, several non-Cd materials, such as ZnSe, InP, Cu-based QDs, and carbon dots, have been investigated in EL devices. ${ }^{98,107,118,123}$ Nevertheless, the EL peaks of these devices are mostly above $480 \mathrm{~nm}$, and the device performance shows a huge margin with conventional QD-LEDs using Cd-based QDs. Therefore, researchers must urgently optimize synthetic methods to synthesize nanocrystals with few defects and uniform sizes as well as device architectures to balance charge injection and transport in devices.

\section{(4) Applications of blue QD-LEDs in lightings and displays}

Currently, there is no precedent for mass production of any solution-processed LEDs. The application of QDs with large-area uniformity and high PL QYs in lightings and displays may require flexible plastic substrates and low manufacturing cost of the QD-LEDs. However, QD-LEDs based on flexible plastic substrates show a huge margin in device performance based on the requirements of lightings and displays, and the flexible plastic substrates introduce additional problems, such as encapsulation. Furthermore, the large-scale synthesis of colloidal QDs has rarely been reported, which increases the manufacturing costs of the QD emitting layer. Extensive efforts should be devoted to finding a highly flexible polymeric film (e.g., polyethylene naphthalate (PEN), polyethylene terephthalate (PET), or polyimide (PI)) and a highly transparent metal electrode (e.g., Ag nanowire or graphene) to realize flexible and transparent devices. ${ }^{129-133}$ Moreover, new synthesis methods should be developed to synthesize blue QD emitting materials on a large scale.

\section{(5) Perspective}

Finally, because they are similar to OLEDs, QD-LEDs possess a high freedom of device design, a fascinating aspect for next-generation displays; they can even rival OLEDs and micro-LEDs, and we look forward to seeing the successful commercialization of QD-LED displays in the near future.

\section{Conflicts of interest}

The authors declare no competing financial interest.

\section{Acknowledgements}

This work was financially supported by the National Natural Science Foundation of China (Grant No. 61674011, 61922028,
61874039, 61735004 and 61974009, 51802079), the Key Project of National Natural Science Foundation of China (Grant No. U1604261), and the Innovation Research Team of Science and Technology in Henan Province (20IRTSTHN020).

\section{References}

1 M. G. Bawendi, M. L. Steigerwald and L. E. Brus, The quantum mechanics of larger semiconductor clusters ("quantum dots"), Annu. Rev. Phys. Chem., 1990, 41, 477.

2 L. Brus, Electronic wave functions in semiconductor clusters: experiment and theory, J. Phys. Chem., 1986, 90, 2555.

3 B. D. Chernomordik, A. R. Marshall, G. F. Pach, J. M. Luther and M. C. Beard, Quantum dot solar cell fabrication protocols, Chem. Mater., 2016, 29, 189.

4 Y. L. Lee and Y. S. Lo, Highly efficient quantum-dotsensitized solar cell based on co-sensitization of CdS/CdSe, Adv. Funct. Mater., 2009, 19, 604.

5 B. R. Smith and S. S. Gambhir, Nanomaterials for in vivo imaging, Chem. Rev., 2017, 117, 901.

6 J. Gao, S. C. Nguyen, N. D. Bronstein and A. P. Alivisatos, Solution processed, high-speed, and high-quantum-efficiency quantum dot infrared photodetectors, ACS Photonics, 2016, 3, 1217.

7 K. Qiao, H. Deng, X. Yang, D. Dong, M. Li, L. Hu, H. Liu, $\mathrm{H}$. Song and J. Tang, Spectra-selective PbS quantum dot infrared photodetectors, Nanoscale, 2016, 8, 7137.

8 S. Jun and E. Jang, Interfused semiconductor nanocrystals: brilliant blue photoluminescence and electroluminescence, Chem. Commun., 2005, 4616.

9 X. Dai, Y. Deng, X. Peng and Y. Jin, Quantum-dot lightemitting diodes for large-area displays: towards the dawn of commercialization, Adv. Mater., 2017, 29, 1607022.

10 H. Moon, C. Lee, W. Lee, J. Kim and H. Chae, Stability of quantum dots, quantum dot films, and quantum dot lightemitting diodes for display applications, Adv. Mater., 2019, 1804294.

11 F. Chen, Z. Guan and A. Tang, Nanostructure and device architecture engineering for high-performance quantumdot light-emitting diodes, J. Mater. Chem. C, 2018, 6, 10958.

12 X. Dai, Z. Zhang, Y. Jin, Y. Niu, H. Cao, X. Liang, L. Chen, J. Wang and X. Peng, Solution-processed, high-performance lightemitting diodes based on quantum dots, Nature, 2014, 515, 96.

13 X. Li, Q. Lin, J. Song, H. Shen, H. Zhang, L. S. Li, X. Li and Z. Du, Quantum-dot light-emitting diodes for outdoor displays with high stability at high brightness, Adv. Opt. Mater., 2019, 1901145.

14 P. Shen, F. Cao, H. Wang, B. Wei, F. Wang, X. W. Sun and $\mathrm{X}$. Yang, Solution-processed double-junction quantum-dot light-emitting diodes with an EQE of over 40\%, ACS Appl. Mater. Interfaces, 2018, 11, 1065.

15 Q. Su, H. Zhang, F. Xia, X. W. Sun and S. Chen, Tandem red quantum-dot light-emitting diodes with external quantum efficiency over 34\%, SID Int. Symp. Dig. Tech. Pap., 2018, 49, 977. 
16 K. P. Acharya, A. Titov, J. Hyvonen, C. Wang, J. Tokarz and P. H. Holloway, High efficiency quantum dot light emitting diodes from positive aging, Nanoscale, 2017, 9, 14451.

17 Q. Su, Y. Sun, H. Zhang and S. Chen, Origin of positive aging in quantum-dot light-emitting diodes, Adv. Sci., 2018, 5, 1800549.

18 Q. Su, H. Zhang, Y. Sun, X. W. Sun and S. Chen, Enhancing the performance of quantum-dot light-emitting diodes by postmetallization annealing, ACS Appl. Mater. Interfaces, 2018, 10, 23218.

19 H. Shen, Q. Gao, Y. Zhang, Y. Lin, Q. Lin, Z. Li, L. Chen, Z. Zeng, X. Li, Y. Jia, S. Wang, Z. Du, L. S. Li and Z. Zhang, Visible quantum dot light-emitting diodes with simultaneous high brightness and efficiency, Nat. Photonics, 2019, 13, 192.

20 Y. Yang, Y. Zheng, W. Cao, A. Titov, J. Hyvonen, J. R. Manders, J. Xue, P. H. Holloway and L. Qain, High-efficiency light-emitting devices based on quantum dots with tailored nanostructures, Nat. Photonics, 2015, 9, 259.

21 Z. Li, Y. Hu, H. Shen, Q. Lin, L. Wang, H. Wang, W. Zhao and L. S. Li, Efficient and long-life green light-emitting diodes comprising tridentate thiol capped quantum dots, Laser Photonics Rev., 2017, 11, 1600227.

22 K. Lin, J. Xing, L. N. Quan, F. P. G. de Arquer, X. Gong, J. Lu, L. Xie, W. Zhao, D. Zhang, C. Yan, W. Li, X. Liu, Y. Lu, J. Kirman, E. H. Sargent, Q. Xiong and Z. Wei, Perovskite light-emitting diodes with external quantum efficiency exceeding 20 percent, Nature, 2018, 562, 245.

23 T. Chiba, Y. Hayashi, H. Ebe, K. Hoshi, J. Sato, S. Sato, Y. J. Pu, S. Ohisa and J. Kido, Anion-exchange red perovskite quantum dots with ammonium iodine salts for highly efficient light-emitting devices, Nat. Photonics, 2018, 12, 681.

24 Z. Wang, X. Zhang, W. Xin, D. Yao, Y. Liu, L. Zhang, W. Liu, W. Zhang, W. Zheng, B. Yang and H. Zhang, Facile synthesis of $\mathrm{Cu}-\mathrm{In}-\mathrm{S} / \mathrm{ZnS}$ core/shell quantum dots in 1-dodecanethiol for efficient light-emitting diodes with an external quantum efficiency of 7.8\%, Chem. Mater., 2018, 30, 8939.

25 Y. Li, X. Hou, X. Dai, Z. Yao, L. Lv, Y. Jin and X. Peng, Stoichiometry-controlled InP-based quantum dots: synthesis, photoluminescence, and electroluminescence, J. Am. Chem. Soc., 2019, 141, 6448.

26 H. Moon, W. Lee, J. Kim, D. Lee, S. Cha, S. Shin and $\mathrm{H}$. Chae, Composition-tailored ZnMgO nanoparticles for electron transport layers of highly efficient and bright InPbased quantum dot light emitting diodes, Chem. Commun., 2019, 55, 13299.

27 P. O. Anikeeva, C. F. Madigan, J. E. Halpert, M. G. Bawendi and V. Bulović, Electronic and excitonic processes in lightemitting devices based on organic materials and colloidal quantum dots, Phys. Rev. B, 2008, 78, 085434.

28 Y. Zhang, F. Zhang, H. Wang, L. Wang, F. Wang, Q. Lin, H. Shen and L. S. Li, High-efficiency CdSe/CdS nanorod-based red light-emitting diodes, Opt. Express, 2019, 27, 7935.

29 S. Chen, W. Cao, T. Liu, S. W. Tsang, Y. Yang, X. Yan and L. Qian, On the degradation mechanisms of quantum-dot light-emitting diodes, Nat. Commun., 2019, 10, 765.
30 Z. Tan, F. Zhang, T. Zhu and J. Xu, Bright and colorsaturated emission from blue light-emitting diodes based on solution-processed colloidal nanocrystal quantum dots, Nano Lett., 2007, 7, 3803.

31 P. O. Anikeeva, J. E. Halpert, M. G. Bawendi and V. Bulović, Quantum dot light-emitting devices with electroluminescence tunable over the entire visible spectrum, Nano Lett., 2009, 9, 2532.

32 L. Qian, Y. Zheng, J. Xue and P. H. Holloway, Stable and efficient quantum-dot light-emitting diodes based on solution-processed multilayer structures, Nat. Photonics, 2011, 5, 543.

33 L. Wang, J. Lin, Y. Hu, X. Guo, Y. Lv, Z. Tang, J. Zhao, Y. Fan, N. Zhang, Y. Wang and X. Liu, Blue quantum dot light-emitting diodes with high electroluminescent efficiency, ACS Appl. Mater. Interfaces, 2017, 9, 38755.

34 H. Shen, S. Wang, H. Wang, J. Niu, L. Qian, Y. Yang, A. Titov, J. Hyvonen, Y. Zheng and L. S. Li, Highly efficient blue-green quantum dot light-emitting diodes using stable low-cadmium quaternary-alloy ZnCdSSe/ZnS core/shell nanocrystals, ACS Appl. Mater. Interfaces, 2013, 5, 4260.

35 K. H. Lee, J. H. Lee, W. S. Song, H. Ko, C. Lee, J. H. Lee and H. Yang, Highly efficient, color-pure, color-stable blue quantum dot light-emitting devices, ACS Nano, 2013, 7, 7295.

36 H. Shen, X. Bai, A. Wang, H. Wang, L. Qian, Y. Yang, A. Titov, J. Hyvonen, Y. Zheng and L. S. Li, High-efficient deep-blue light-emitting diodes by using high quality $\mathrm{Zn}_{x} \mathrm{Cd}_{1-x} \mathrm{~S} / \mathrm{ZnS}$ core/shell quantum dots, Adv. Funct. Mater., 2014, 24, 2367.

37 I. Cho, H. Jung, B. G. Jeong, D. Hahm, J. H. Chang, T. Lee, K. Char, D. C. Lee, J. Lim, C. Lee, J. Cho and W. K. Bae, Ligand-asymmetric janus quantum dots for efficient bluequantum dot light-emitting diodes, ACS Appl. Mater. Interfaces, 2018, 10, 22453.

38 H. Shen, W. Cao, N. T. Shewmon, C. Yang, L. S. Li and J. Xue, High-efficiency, low turn-on voltage blue-violet quantum-dot-based light-emitting diodes, Nano Lett., 2015, 15, 1211.

39 H. Shen, Q. Lin, W. Cao, C. Yang, N. T. Shewmon, H. Wang, J. Niu, L. S. Li and J. Xue, Efficient and long-lifetime full-color light-emitting diodes using high luminescence quantum yield thick-shell quantum dots, Nanoscale, 2017, 9, 13583.

40 O. Wang, L. Wang, Z. Li, Q. Xu, Q. Lin, H. Wang, Z. Du, H. Shen and L. S. Li, High-efficiency, deep blue ZnCdS/ $\mathrm{Cd}_{x} \mathrm{Zn}_{1-x} \mathrm{~S} / \mathrm{ZnS}$ quantum-dot-light-emitting devices with an EQE exceeding 18\%, Nanoscale, 2018, 10, 5650.

41 Q. Lin, L. Wang, Z. Li, H. Shen, L. Guo, Y. Kuang, H. Wang and L. S. Li, Nonblinking quantum-dot-based blue lightemitting diodes with high efficiency and a balanced charge-injection process, ACS Photonics, 2018, 5, 939.

42 G. J. Supran, Y. Shirasaki, K. W. Song, J. M. Caruge, P. T. Kazlas, S. Coe-Sullivan, T. L. Andrew, M. G. Bawendi and V. Bulović, QLEDs for displays and solid-state lighting, MRS Bull., 2013, 38, 703.

43 F. Wang, S. Jin, W. Sun, J. Lin, B. You, Y. Li, B. Zhang, T. Hayat, A. Alsaedi and Z. Tan, Enhancing the performance 
of blue quantum dots light-emitting diodes through interface engineering with deoxyribonucleic acid, $A d v$. Opt. Mater., 2018, 6, 1800578.

44 J. Li, Z. Liang, Q. Su, H. Jin, K. Wang, G. Xu and X. Xu, Small molecule-modified hole transport layer targeting low turn-on-voltage, bright, and efficient full-color quantum dot light emitting diodes, ACS Appl. Mater. Interfaces, 2018, 10, 3865.

45 F. Wang, W. Sun, P. Liu, Z. Wang, J. Zhang, J. Wei, Y. Li, T. Hayat, A. Alsaedi and Z. Tan, Achieving balanced charge injection of blue quantum dots light-emitting diodes through transport layer doping strategies, J. Phys. Chem. Lett., 2019, 10, 960.

46 X. Qu, N. Zhang, R. Cai, B. Kang, S. Chen, B. Xu, K. Wang and $\mathrm{X}$. W. Sun, Improving blue quantum dot light-emitting diodes by a lithium fluoride interfacial layer, ACS Appl. Phys. Lett., 2019, 114, 071101.

47 D. Li, J. Bai, T. Zhang, C. Chang, X. Jin, Z. Huang, B. Xu and Q. Li, Blue quantum dot light-emitting diodes with high luminance by improving the charge transfer balance, Chem. Commun., 2019, 55, 3501.

48 Y. Sun, W. Wang, H. Zhang, Q. Su, J. Wei, P. Liu, S. Chen and S. Zhang, High-performance quantum dot light-emitting diodes based on Al-doped $\mathrm{ZnO}$ nanoparticles electron transport layer, ACS Appl. Mater. Interfaces, 2018, 10, 18902.

49 T. Cheng, F. Wang, W. Sun, Z. Wang, J. Zhang, B. You, Y. Li, T. Hayat, A. Alsaed and Z. Tan, High-performance blue quantum dot light-emitting diodes with balanced charge injection, Adv. Electron. Mater., 2019, 5, 1800794.

50 J. Kwak, W. K. Bae, D. Lee, I. Park, J. Lim, M. Park, H. Cho, H. Woo, D. Y. Yoon, K. Char, S. Lee and C. Lee, Bright and efficient full-color colloidal quantum dot light-emitting diodes using an inverted device structure, Nano Lett., 2012, 12, 2362.

51 Z. Zhong, J. Zou, C. Jiang, L. Lan, C. Song, Z. He, L. Mu, J. Wang, J. Peng and Y. Cao, Improved color purity and efficiency of blue quantum dot light-emitting diodes, Org. Electron., 2018, 58, 245.

52 A. Castan, H. M. Kim and J. Jang, All-solution-processed inverted quantum-dot light-emitting diodes, ACS Appl. Mater. Interfaces, 2014, 6, 2508.

53 Y. Liu, C. Jiang, C. Song, J. Wang, L. Mu, Z. He, Z. Zhong, Y. Cun, C. Mai, J. Wang, J. Peng and Y. Cao, Highly efficient all-solution processed inverted quantum dots based light emitting diodes, ACS Nano, 2018, 12, 1564.

54 H. Zhang, S. Chen and X. W. Sun, Efficient red/green/blue tandem quantum-dot light-emitting diodes with external quantum efficiency exceeding 21\%, ACS Nano, 2017, 12, 697.

55 W. Ji, T. Wang, B. Zhu, H. Zhang, R. Wang, D. Zhang, L. Chen, Q. Yang and H. Zhang, Highly efficient flexible quantum-dot light emitting diodes with an ITO/Ag/ITO cathode, J. Mater. Chem. C, 2017, 5, 4543.

56 Z. K. Tan, R. S. Moghaddam, M. L. Lai, P. Docampo, R. Higler, F. Deschler, M. Price, A. Sadhanala, L. M. Pazos, D. Credgington, F. Hanusch, T. Bein, H. J. Snaith and R. H. Friend, Bright light-emitting diodes based on organometal halide perovskite, Nat. Nanotechnol., 2014, 9, 687.
57 X. Yang, X. Zhang, J. Deng, Z. Chu, Q. Jiang, J. Meng, P. Wang, L. Zhang, Z. Yin and J. You, Efficient green lightemitting diodes based on quasi-two-dimensional composition and phase engineered perovskite with surface passivation, Nat. Commun., 2018, 9, 570.

58 J. Chang, S. Zhang, N. Wang, Y. Sun, Y. Wei, R. Li, C. Yi, J. Wang and W. Huang, Enhanced performance of red perovskite light-emitting diodes through the dimensional tailoring of perovskite multiple quantum wells, J. Phys. Chem. Lett., 2018, 9, 881.

59 L. Protesescu, S. Yakunin, M. I. Bodnarchuk, F. Krieg, R. Caputo, C. H. Hendon, R. X. Yang, A. Walsh and V. Kovalenko, Nanocrystals of cesium lead halide perovskites $\left(\mathrm{CsPbX}_{3}, \mathrm{X}=\mathrm{Cl}, \mathrm{Br}\right.$, and I): novel optoelectronic materials showing bright emission with wide color gamut, Nano Lett., 2015, 15, 3692.

60 S. Yuan, Z. K. Wang, M. P. Zhuo, Q. S. Tian, Y. Jin and L. S. Liao, Self-assembled high quality $\mathrm{CsPbBr}_{3}$ quantum dot films toward highly efficient light-emitting diodes, $A C S$ Nano, 2018, 12, 9541.

61 G. Li, J. Huang, H. Zhu, Y. Li, J. X. Tang and Y. Jiang, Surface ligand engineering for near-unity quantum yield inorganic halide perovskite QDs and high-performance QLEDs, Chem. Mater., 2018, 30, 6099.

62 Q. Van Le, H. W. Jang and S. Y. Kim, Recent advances toward high-efficiency halide perovskite light-emitting diodes: review and perspective, Small Methods, 2018, 2, 1700419.

63 N. K. Kumawat, X. K. Liu, D. Kabra and F. Gao, Blue perovskite light-emitting diodes: progress, challenges and future directions, Nanoscale, 2019, 11, 2109.

64 Y. Cao, N. Wang, H. Tian, J. Guo, Y. Wei, H. Chen, Y. Miao, W. Zou, K. Pan, Y. He, H. Cao, Y. Ke, M. Xu, Y. Wang, M. Yang, K. Du, Z. Fu, D. Kong, D. Dai, Y. Jin, G. Li, H. Li, Q. Peng, J. Wang and W. Huang, Perovskite light-emitting diodes based on spontaneously formed submicrometrescale structures, Nature, 2018, 562, 249.

65 N. K. Kumawat, A. Dey, A. Kumar, S. P. Gopinathan, K. L. Narasimhan and D. Kabra, Band gap tuning of $\mathrm{CH}_{3} \mathrm{NH}_{3} \mathrm{~Pb}\left(\mathrm{Br}_{1-x} \mathrm{Cl}_{x}\right)_{3}$ hybrid perovskite for blue electroluminescence, ACS Appl. Mater. Interfaces, 2015, 7, 13119.

66 H. P. Kim, J. Kim, B. S. Kim, H. M. Kim, J. Kim, A. R. M. Yusoff, J. Jang and M. K. Nazeeruddin, High-efficiency, blue, green, and near-infrared light-emitting diodes based on triple cation perovskite, Adv. Opt. Mater., 2017, 5, 1600920.

67 F. Zhang, C. Xiao, Y. Li, X. Zhang, J. Tang, S. Chang, Q. Pei and H. Zhong, Gram-scale synthesis of blue-emitting $\mathrm{CH}_{3} \mathrm{NH}_{3} \mathrm{PbBr}_{3}$ quantum dots through phase transfer strategy, Front. Chem., 2018, 6, 444.

68 D. Liang, Y. Peng, Y. Fu, M. J. Shearer, J. Zhang, J. Zhai, J. Zhang, R. J. Hamers, T. L. Andrew and S. Jin, Color-pure violet-light-emitting diodes based on layered lead halide perovskite nanoplates, ACS Nano, 2016, 10, 6897.

69 L. Cheng, Y. Cao, R. Ge, Y. Q. Wei, N. N. Wang, J. P. Wang and W. Huang, Sky-blue perovskite light-emitting diodes based on quasi-two-dimensional layered perovskites, Chin. Chem. Lett., 2017, 28, 29. 
70 D. Yang, Y. Zou, P. Li, Q. Liu, L. Wu, H. Hu, Y. Xu, B. Sun, Q. Zhang and S. T. Lee, Large-scale synthesis of ultrathin cesium lead bromide perovskite nanoplates with precisely tunable dimensions and their application in blue lightemitting diodes, Nano Energy, 2018, 47, 235.

71 Z. Yuan, Y. Shu, Y. Xin and B. Ma, Highly luminescent nanoscale quasi-2D layered lead bromide perovskites with tunable emissions, Chem. Commun., 2016, 52, 3887.

72 S. Kumar, J. Jagielski, S. Yakunin, P. Rice, Y. C. Chiu, M. Wang, G. Nedelcu, Y. Kim, S. Lin, E. J. G. Santos, M. V. Kovalenko and C. J. Shin, Efficient blue electroluminescence using quantum-confined two-dimensional perovskites, ACS Nano, 2016, 10, 9720.

73 Y. Jiang, C. Qin, M. Cui, T. He, K. Liu, Y. Huang, M. Luo, L. Zhang, H. Xu, S. Li, J. Wei, Z. Liu, H. Wang, G. H. Kim, M. Yuan and J. Chen, Spectra stable blue perovskite lightemitting diodes, Nat. Commun., 2019, 10, 1868.

74 Z. Chen, C. Zhang, X. F. Jiang, M. Liu, R. Xia, T. Shi, D. Chen, Q. Xue, Y. J. Zhao, S. Su, H. L. Yip and Y. Cao, High-performance color-tunable perovskite light emitting devices through structural modulation from bulk to layered film, Adv. Mater., 2017, 29, 1603157.

75 Q. Wang, J. Ren, X. F. Peng, X. X. Ji and X. H. Yang, Efficient sky-blue perovskite light-emitting devices based on ethylammonium bromide induced layered perovskites, ACS Appl. Mater. Interfaces, 2017, 9, 29901.

76 Z. Ren, X. Xiao, R. Ma, H. Lin, K. Wang, X. W. Sun and W. C. H. Choy, Hole Transport Bilayer Structure for Quasi-2D Perovskite Based Blue Light-Emitting Diodes with High Brightness and Good Spectral Stability, Adv. Funct. Mater., 2019, 29, 1905339.

77 J. Xing, Y. Zhao, M. Askerka, L. N. Quan, X. Gong, W. Zhao, J. Zhao, H. Tan, G. Long, L. Gao, Z. Yang, O. Voznyy, J. Tang, Z. H. Lu, Q. Xiong and E. H. Sargent, Colorstable highly luminescent sky-blue perovskite lightemitting diodes, Nat. Commun., 2018, 9, 3541.

78 P. Vashishtha, M. Ng, S. B. Shivarudraiah and J. E. Halpert, High efficiency blue and green light-emitting diodes using ruddlesden-popper inorganic mixed halide perovskites with butylammonium interlayers, Chem. Mater., 2018, 31, 83.

79 Z. Li, Z. Chen, Y. Yang, Q. Xue, H. L. Yip and Y. Cao, Modulation of recombination zone position for quasi-twodimensional blue perovskite light-emitting diodes with efficiency exceeding 5\%, Nat. Commun., 2019, 10, 1027.

80 Y. Liu, J. Cui, K. Du, H. Tian, Z. He, Q. Zhou, Z. Yang, Y. Deng, D. Chen, X. Zou, Y. Ren, L. Wang, H. Zhu, B. Zhao, D. Di, J. Wang, R. H. Friend and Y. Jin, Efficient blue lightemitting diodes based on quantum-confined bromide perovskite nanostructures, Nat. Photonics, 2019, 13, 760.

81 Q. Wang, X. Wang, Z. Yang, N. Zhou, Y. Deng, J. Zhao, X. Xiao, P. Rudd, A. Moran, Y. Yan and J. Huang, Efficient sky-blue perovskite light-emitting diodes via photoluminescence enhancement, Nat. Commun., 2019, 10, 1.

82 J. Song, J. Li, X. Li, L. Xu, Y. Dong and H. Zeng, Quantum dot light-emitting diodes based on inorganic perovskite cesium lead halides ( $\left.\mathrm{CsPbX}_{3}\right)$, Adv. Mater., 2016, 27, 7162.
83 E. P. Yao, Z. Yang, L. Meng, P. Sun, S. Dong, Y. Yang and Y. Yang, High-brightness blue and white LEDs based on inorganic perovskite nanocrystals and their composites, Adv. Mater., 2017, 1606859.

84 M. K. Gangishetty, S. Hou, Q. Quan and N. Congreve, Blue perovskite LEDs: reducing architecture limitations for efficient blue perovskite light-emitting diodes, Adv. Mater., 2018, 30, 1706226.

85 J. Pan, L. N. Quan, Y. Zhao, W. Peng, B. Murali, S. P. Sarmah, M. Yuan, L. Sinatra, N. M. Alyami, J. Liu, E. Yassitepe, Z. Yang, O. Voznyy, R. Comin, M. N. Hedhili, O. F. Mohammed, Z. H. Lu, D. H. Kim, E. H. Sargent and O. M. Bakr, Highly efficient perovskite-quantum-dot lightemitting diodes by surface engineering, Adv. Mater., 2016, 28, 8718.

86 P. Vashishtha and J. E. Halpert, Field-driven ion migration and color instability in red-emitting mixed halide perovskite nanocrystal light-emitting diodes, Chem. Mater., 2017, 29, 5965.

87 G. Li, F. W. R. Rivarola, N. J. L. K. Davis, S. Bai, T. C. Jellicoe, F. de la Peña, S. Hou, C. Ducati, F. Gao, R. H. Friend, N. C. Greenham and Z. K. Tan, Highly efficient perovskite nanocrystal light-emitting diodes enabled by a universal crosslinking method, Adv. Mater., 2016, 28, 3528.

88 S. T. Ochsenbein, F. Krieg, Y. Shynkarenko, G. Rainò and M. V. Kovalenko, Engineering color-stable blue light-emitting diodes with lead-halide perovskite nanocrystals, ACS Appl. Mater. Interfaces, 2019, 11, 21655.

89 C. H. A. Li, Z. Zhou, P. Vashishtha and J. E. Halpert, The future is blue (LEDs): why chemistry is the key to perovskite displays, Chem. Mater., 2019, 31, 6003.

90 Z. Liang, S. Zhao, Z. Xu, B. Qiao, P. Song, D. Gao and X. Xu, Shape-controlled synthesis of all-inorganic $\mathrm{CsPbBr}_{3}$ perovskite nanocrystals with bright blue emission, ACS Appl. Mater. Interfaces, 2016, 8, 28824.

91 M. Liu, G. Zhong, Y. Yin, J. Miao, K. Li, C. Wang, X. Xu, C. Shen and H. Meng, Aluminum-doped cesium lead bromide perovskite nanocrystals with stable blue photoluminescence used for display backlight, Adv. Sci., 2017, 1700335.

92 C. Bi, S. Wang, Q. Li, S. V. Kershaw, J. Tian and A. L. Rogach, Thermally stable copper(II) doped cesium lead halide perovskite quantum dots with a strong blue emission, J. Phys. Chem. Lett., 2019, 10, 943.

93 Y. Zhai, X. Bai, G. Pan, J. Zhu, H. Shao, B. Dong, L. Xu and H. Song, Effective blue-violet photoluminescence through lanthanum and fluorine ions co-doping for $\mathrm{CsPbCl}_{3}$ perovskite quantum dots, Nanoscale, 2019, 11, 2484.

94 S. Zou, Y. Liu, J. Li, C. Liu, R. Feng, F. Jiang, Y. Li, J. Song, H. Zeng, M. Hong and X. Chen, Stabilizing cesium lead halide perovskite lattice through $\mathrm{Mn}$ (II) substitution for airstable light-emitting diodes, J. Am. Chem. Soc., 2017, 139, 11443.

95 S. Hou, M. K. Gangishetty, Q. Quan and D. N. Congreve, Efficient blue and white perovskite light-emitting diodes via manganese doping, Joule, 2018, 2, 2421.

96 C. Xiang, W. Koo, S. Chen, F. So, X. Liu, X. Kong and Y. Wang, Solution processed multilayer cadmium-free 
blue/violet emitting quantum dots light emitting diodes, Appl. Phys. Lett., 2012, 101, 053303.

97 W. Ji, P. Jing, W. Xu, X. Yuan, Y. Wang, J. Zhao and A. K. Y. Jen, High color purity $\mathrm{ZnSe} / \mathrm{ZnS}$ core/shell quantum dot based blue light emitting diodes with an inverted device structure, Appl. Phys. Lett., 2013, 103, 053106.

98 A. Wang, H. Shen, S. Zang, Q. Lin, H. Wang, L. Qian, J. Niu and L. S. Li, Bright, efficient, and color-stable violet ZnSebased quantum dot light-emitting diodes, Nanoscale, 2015, 7, 2951.

99 H. Shen, H. Wang, X. Li, J. Z. Niu, H. Wang, X. Chen and L. S. Li, Phosphine-free synthesis of high quality ZnSe, ZnSe/ZnS, and Cu-, Mn-doped ZnSe nanocrystals, Dalton Trans., 2009, 10534.

100 Y. Liu, Y. Tang, Y. Ning, M. Li, H. Zhang and B. Yang, "One pot" synthesis and shape control of ZnSe semiconductor nanocrystals in liquid paraffin, J. Mater. Chem., 2010, 20, 4451.

101 H. Zhang, N. Hu, Z. Zeng, Q. Lin, F. Zhang, A. Tang, Y. Jia, L. S. Li, H. Shen, F. Teng and Z. Du, High-efficiency green InP quantum dot-based electroluminescent device comprising thick-shell quantum dots, Adv. Opt. Mater., 2019, 7, 1801602.

102 E. P. Jang, C. Y. Han, S. W. Lim, J. H. Jo, D. Y. Jo, S. H. Lee, S. Y. Yoon and H. Yang, Synthesis of alloyed ZnSeTe quantum dots as bright, color-pure blue emitters, ACS Appl. Mater. Interfaces, 2019, 11, 46062.

103 S. Koh, T. Eom, W. D. Kim, K. Lee, D. Lee, Y. K. Lee, H. Kim, W. K. Bae and D. C. Lee, Zinc-phosphorus complex working as an atomic valve for colloidal growth of monodisperse indium phosphide quantum dots, Chem. Mater., 2017, 29, 6346.

104 P. Ramasamy, K. J. Ko, J. W. Kang and J. S. Lee, Two-step "seed-mediated" synthetic approach to colloidal indium phosphide quantum dots with high-purity photo- and electroluminescence, Chem. Mater., 2018, 30, 3643.

105 D. Hahm, J. H. Chang, B. G. Jeong, P. Park, J. Kim, S. Lee, J. Choi, W. D. Kim, S. Rhee, J. Lim, D. C. Lee, C. Lee, K. Char and W. K. Bae, Design principle for bright, robust, and color-pure InP/ZnSe $\mathrm{S}_{1-x} / \mathrm{ZnS}$ heterostructures, Chem. Mater., 2019, 31, 3476.

106 S. Tamang, C. Lincheneau, Y. Hermans, S. Jeong and P. Reiss, Chemistry of InP nanocrystal syntheses, Chem. Mater., 2016, 28, 2491.

107 W. Shen, H. Tang, X. Yang, Z. Cao, T. Cheng, X. Wang, Z. Tan, J. You and Z. Deng, Synthesis of highly fluorescent InP/ZnS small-core/thick-shell tetrahedral-shaped quantum dots for blue light-emitting diodes, J. Mater. Chem. C, 2017, 5, 8243.

108 H. Zhang, X. Ma, Q. Lin, Z. Zeng, H. Wang, L. S. Li, H. Shen, Y. Jia and Z. Du, High-brightness blue InP quantum dotsbased electroluminescent devices: the role of shell thickness, J. Phys. Chem. Lett., 2020, 11, 960.

109 X. Yang, D. Zhao, K. S. Leck, S. T. Tan, Y. X. Tang, J. Zhao, H. V. Demir and X. W. Sun, Full visible range covering InP/ ZnS nanocrystals with high photometric performance and their application to white quantum dot light-emitting diodes, Adv. Mater., 2012, 24, 4180.

110 J. P. Park, J. J. Lee and S. W. Kim, Highly luminescent InP/ $\mathrm{GaP} / \mathrm{ZnS}$ QDs emitting in the entire color range via a heating up process, Sci. Rep., 2016, 6, 30094.

111 Y. Altıntas, M. Y. Talpur, M. Ünlü and E. Mutlugün, Highly efficient Cd-free alloyed core/shell quantum dots with optimized precursor concentrations, J. Phys. Chem. C, 2016, 120, 7885.

112 K. D. Wegner, S. Pouget, W. L. Ling, M. Carrière and P. Reiss, Gallium-A versatile element for tuning the photoluminescence properties of InP quantum dots, Chem. Commun., 2019, 55, 1663.

113 P. Ramasamy, N. Kim, Y. S. Kang, O. Ramirez and J. S. Lee, Tunable, bright, and narrow-band luminescence from colloidal indium phosphide quantum dots, Chem. Mater., 2017, 29, 6893.

114 K. Lim, H. S. Jang and K. Woo, Synthesis of blue emitting $\mathrm{InP} / \mathrm{ZnS}$ quantum dots through control of competition between etching and growth, Nanotechnol., 2012, 23, 485609.

115 J. H. Kim, K. H. Lee, D. Y. Jo, Y. Lee, J. Y. Hwang and H. Yang, Cu-In-Ga-S quantum dot composition-dependent device performance of electrically driven light-emitting diodes, Appl. Phys. Lett., 2014, 105, 133104.

116 B. Y. Kim, J. H. Kim, K. H. Lee, E. P. Jang, C. Y. Han, J. H. Jo, H. S. Jang and H. Yang, Synthesis of highly efficient azure-to-blue-emitting Zn-Cu-Ga-S quantum dots, Chem. Commun., 2017, 53, 4088.

117 J. H. Kim, K. H. Kim, S. Y. Yoon, Y. Kim, S. H. Lee, H. S. Kim and H. Yang, Tunable emission of bluish Zn-Cu-Ga-S quantum dots by Mn doping and their electroluminescence, ACS Appl. Mater. Interfaces, 2019, 11, 8250.

118 S. Y. Yoon, J. H. Kim, K. H. Kim, C. Y. Han, J. H. Jo, D. Y. Jo, S. Hong, J. Y. Hwang, Y. R. Do and H. Yang, High-efficiency blue and white electroluminescent devices based on nonCd I-III-VI quantum dots, Nano Energy, 2019, 63, 103869.

119 Y. P. Sun, B. Zhou, Y. Lin, W. Wang, K. A. S. Fernando, P. Pathak, M. J. Meziani, B. A. Harruff, X. Wang, H. Wang, P. G. Luo, H. Yang, M. E. Kose, B. Chen, L. M. Veca and S. Y. Xie, Quantum-sized carbon dots for bright and colorful photoluminescence, J. Am. Chem. Soc., 2006, 128, 7756.

120 X. Zhang, Y. Zhang, Y. Wang, S. Kalytchuk, S. V. Kershaw, Y. Wang, P. Wang, T. Zhang, Y. Zhao, H. Zhang, T. Cui, Y. Wang, J. Zhao, W. W. Yu and A. L. Rogach, Colorswitchable electroluminescence of carbon dot light-emitting diodes, ACS Nano, 2013, 7, 11234.

121 F. Yuan, Z. Wang, X. Li, Y. Li, Z. Tan, L. Fan and S. Yang, Bright multicolor bandgap fluorescent carbon quantum dots for electroluminescent light-emitting diodes, $A d v$. Mater., 2017, 29, 1604436.

$122 \mathrm{~J} . \mathrm{Xu}, \mathrm{Y}$. Miao, J. Zheng, Y. Yang and X. Liu, Ultrahigh brightness carbon dot-based blue electroluminescent LEDs by host-guest energy transfer emission mechanism, $A d v$. Opt. Mater., 2018, 6, 1800181.

123 F. Yuan, T. Yuan, L. Sui, Z. Wang, Z. Xi, Y. Li, X. Li, L. Fan, Z. Tan, A. Chen, M. Jin and S. Yang, Engineering triangular 
carbon quantum dots with unprecedented narrow bandwidth emission for multicolored LEDs, Nat. Commun., 2018, 9, 2249.

124 F. Yuan, Y. K. Wang, G. Sharma, Y. Dong, X. Zheng, P. Li, A. Johnston, G. Bappi, J. Z. Fan, H. Kung, B. Chen, M. I. Saidaminov, K. Singh, O. Voznyy, O. M. Bakr, Z. H. Lu and E. H. Sargent, Bright high-colour-purity deep-blue carbon dot light-emitting diodes via efficient edge amination, Nat. Photonics, 2019, 1.

125 W. Deng, X. Jin, Y. Lv, X. Zhang, X. Zhang and J. Jie, 2D ruddlesden-popper perovskite nanoplate based deep-blue light-emitting diodes for light communication, Adv. Funct. Mater., 2019, 29, 1903861.

126 J. Song, O. Wang, H. Shen, Q. Lin, Z. Li, L. Wang, X. Zhang and L. S. Li, Over $30 \%$ external quantum efficiency light-emitting diodes by engineering quantum dot-assisted energy level match for hole transport layer, Adv. Funct. Mater., 2019, 1808377.

127 Z. Yang, Q. Wu, G. Lin, X. Zhou, W. Wu, X. Yang, J. Zhang and W. Li, All-solution processed inverted green quantum dot light-emitting diodes with concurrent high efficiency and long lifetime, Mater. Horiz., 2019, 6, 2009.

128 H. Zhang, F. Wang, Y. Kuang, Z. Li, Q. Lin, H. Shen, H. Wang, L. Guo and L. S. Li, Se/S ratio-dependent properties and application of gradient alloyed $\mathrm{CdSe}_{1-x} \mathrm{~S}_{x}$ quantum dots: shell-free structure, non-blinking photoluminescence with single-exponential decay, and efficient QLEDs, ACS Appl. Mater. Interfaces, 2019, 11, 6238.

129 D. Kim, Y. Fu, S. Kim, W. Lee, K. H. Lee, H. K. Chung, H. J. Lee, H. Yang and H. Chae, Polyethylenimine ethoxylatedmediated all-solution-processed high-performance flexible inverted quantum dot-light-emitting device, ACS Nano, 2017, 11, 1982.

130 J. H. Kim, D. Y. Jo, K. H. Lee, E. P. Jang, C. Y. Han, J. H. Jo and H. Yang, White electroluminescent lighting device based on a single quantum dot emitter, Adv. Mater., 2016, 28, 5093.

131 M. K. Choi, J. Yang, K. Kang, D. C. Kim, C. Choi, C. Park, S. J. Kim, S. I. Chae, T. H. Kim, J. H. Kim, T. Hyeon and D. H. Kim, Wearable red-green-blue quantum dot lightemitting diode array using high-resolution intaglio transfer printing, Nat. Commun., 2015, 6, 7149.

132 C. Y. Han, K. H. Lee, M. S. Kim, J. W. Shin, J. S. Kim, J. H. Hwang, T. Kim, M. S. Oh, J. Kim, Y. R. Do and H. Yang, Solution-processed fabrication of highly transparent mono-and tri-colored quantum dot-light-emitting diodes, Org. Electron., 2017, 45, 145.

133 P. T. Jing, W. Ji, Q. H. Zeng, D. Li, S. N. Qu, J. Wang and D. Zhang, Vacuum-free transparent quantum dot lightemitting diodes with silver nanowire cathode, Sci. Rep., 2015, 5, 12499. 